\title{
Epoxides and aziridines - A mini review
}

\author{
Albert Padwa $^{\mathrm{a} *}$ and S. Shaun Murphree ${ }^{\mathrm{b}}$ \\ ${ }^{a}$ Emory University, Atlanta, GA 30322 \\ E-mail: chemap@emory.edu \\ ${ }^{b}$ Allegheny College, Meadville, PA 16335 \\ E-mail: smurphre@allegheny.edu
}

\section{Dedicated to Professor Jim Coxon on the occasion of his 65th anniversary}

\begin{abstract}
Three-membered heterocyclic rings offer an uncommon combination of reactivity, synthetic flexibility, and atom economy. This mini review surveys a selection of the most recent advances amongst epoxides and aziridines. Emphasis is given to novel synthetic methods and new insights into existing methodologies for the selective construction and controlled reaction of the title compounds reported in the past year's literature. While not the exclusive focus of this minireview, well-represented themes in the literature include asymmetric techniques and environmentally compatible reagents.
\end{abstract}

Keywords: Epoxides, aziridines, three-membered heterocycles, review, enantioselective, synthesis

\section{Table of Contents}

Introduction

Epoxides

Preparation of Epoxides

Metal Mediated Epoxidations

Asymmetric Epoxidations

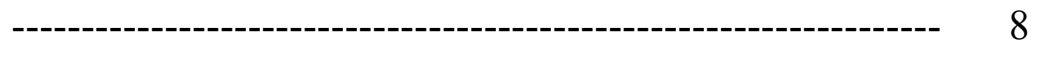

Reaction of Epoxides

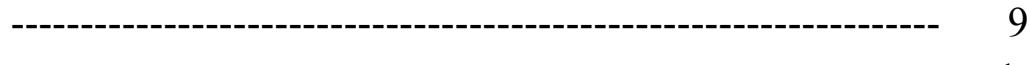

Ring Opening Reactions

Aziridines

Preparation of Aziridines

Reaction of Aziridines

References

\footnotetext{
* Author to whom inquiries concerning this article should be sent
} 


\section{Introduction}

Three-membered heterocycles are invested with a special allure that is derived from their apparent simplicity and spartan architecture. Yet these systems are multifaceted, and the literature continues to provide evidence of their diversity, both in terms of preparative routes and subsequent transformations. These smallest of heterocycles also exhibit a synthetically very useful balance between stability and reactivity. Thus, they are often employed as versatile and selective intermediates. With the potential to introduce two adjacent chiral centers with high atom economy, this methodology rightly deserves a place of prominence in synthetic organic chemistry. The utility of epoxides, for example, in the enantioselective synthesis of oxygensubstituted quaternary carbon centers has been the subject of a recent review. The following is a sampling of highlights from the past year's literature with an emphasis on synthetic utility.

\section{Epoxides}

\section{Preparation of Epoxides}

An important preparative methodology which has developed rapidly over the last few years is the (salen)Mn mediated epoxidation of alkenes (the Jacobsen-Katsuki epoxidation). While the practical utility of this protocol is indisputable, the mechanistic underpinnings have been the matter of some debate. Adding to this ongoing dialectic is a result from a recently published theoretical study, which suggests the salen ligand itself is involved in the transition state of the epoxidation. In one outcome on a rather complex reaction surface, a covalent bond is formed beween the carbon atom of the substrate and the oxygen atom of the salen framework. ${ }^{2}$

A novel immobilized (salen)Mn catalyst has been constructed by incorporating a chiral sulfonato-salen complex into a zinc-aluminum layered double hydroxide (LDH) host. The active catalyst is proposed to exist as an intercalated species spanning the interlayer space (i.e., 1). The complex was shown to promote the selective epoxidation of limonene (2) with molecular oxygen at room temperature in the presence of pivaldehyde and $N$-methylimidazole $(N-\mathrm{MeI})$ under Mukaiyama conditions. The activity of the catalyst is stable even after multiple cycles, and no leaching of manganese has been observed. ${ }^{3}$ Recent mechanistic studies suggest that the epoxidation proceeds via the in situ formation of a peracid (i.e., perpivalic acid) through autoxidation of the aldehyde, which subsequently serves as an oxygen donor during the epoxidation. $^{4}$ 


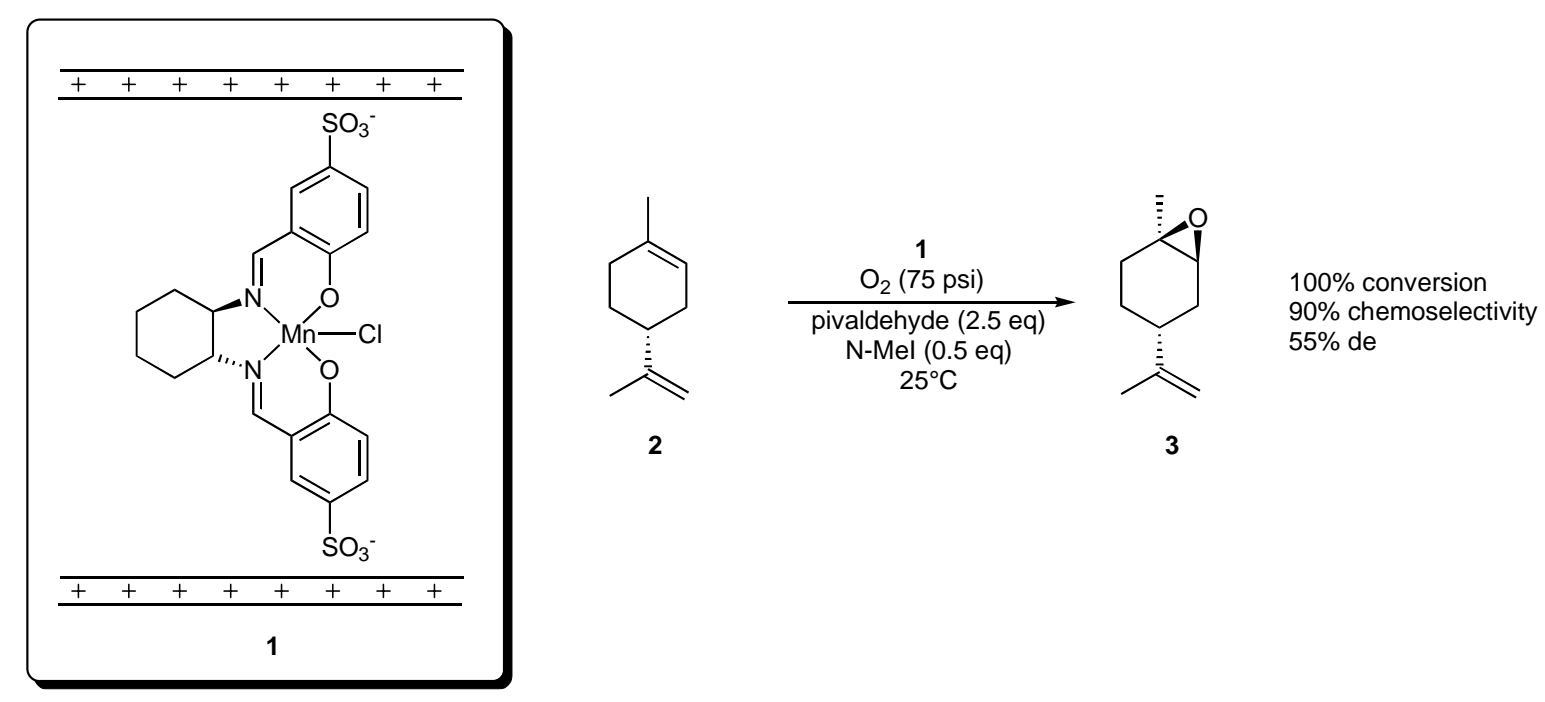

Metal mediated epoxidation is remarkably diverse, with many types of ligand systems being represented. For example, a cytochrome P450 BM-3 mutant (139-3) has been developed using directed evolution, which exhibits high activity towards epoxidation of several non-natural substrates. Thus, exposure of styrene (4) to BM-3 variant 139-3 in phosphate buffer containing methanol and NADPH resulted in the quantitative conversion to styrene oxide (5). For terminal aliphatic alkenes, however, allylic hydroxylation is the predominant process. ${ }^{5}$

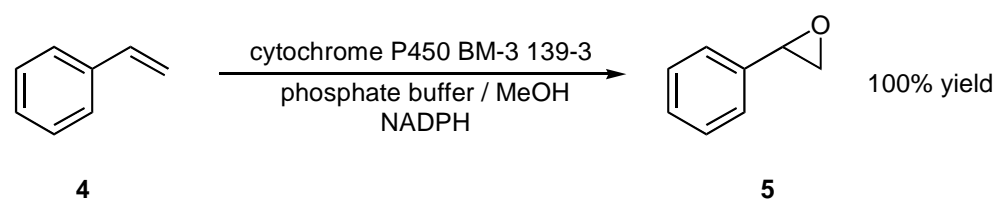

Highly efficient small molecular P450 mimics have also been developed. The sterically stabilized metalloporphyrin [Mn(TDCPP)Cl] (6) catalyzes the mild and highly diastereoselective epoxidation of protected cyclohexenol derivative 7 using hydrogen peroxide as the terminal oxidant. The preference for trans-epoxides is rationalized on the basis of non-bonded interactions between the allylic substituent on the substrate and the bulky porphyrin ligand; diastereoselectivies increase with steric demand of the substituent. ${ }^{6}$ The same metalloporphyrin has been shown to catalyze the epoxidation of aromatic substrates, which has implications not only in the realm of synthetic methodology, but also with respect to the toxicology of environmental polycyclic aromatic hydrocarbons. Thus, phenanthrene (9) is converted to epoxide 10 in excellent yield in three hours with a catalyst loading of $0.3 \mathrm{~mol} \%{ }^{7}$ 

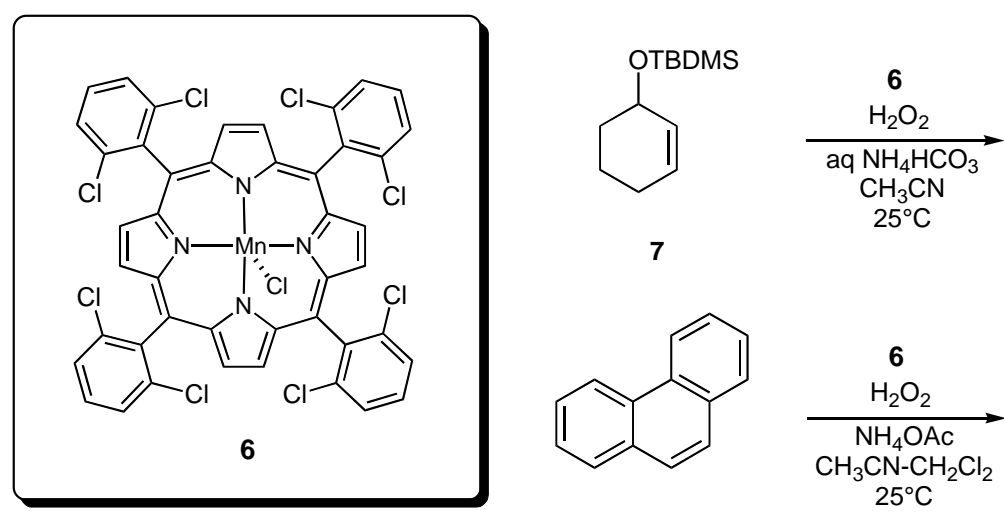<smiles>CCCCOC1CCC[C@@H]2O[C@H]12</smiles>

$100 \%$ conversion

$88 \%$ yield

$33: 1$ trans/cis<smiles>c1ccc2ccccc2c1</smiles>

9

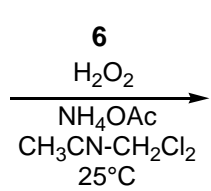
$25^{\circ} \mathrm{C}$<smiles>c1ccc2c(c1)-c1ccccc1C1OC21</smiles>

10

Conventional wisdom holds that these epoxidation reactions occur via oxygen atom transfer directly from the catalyst via the metal-oxo species. As a result of their studies of the structurally similar Mn-corroles (11), Goldberg and co-workers have proposed that at least a secondary pathway might exist in which the metal-oxo species activates the terminal oxidant through simple Lewis acid catalysis (cf. 12). This hypothesis, which is supported by ${ }^{18} \mathrm{O}$ labeling experiments, would explain the long-recognized but poorly understood impact of the terminal oxidant on the course of these epoxidations. ${ }^{8}$

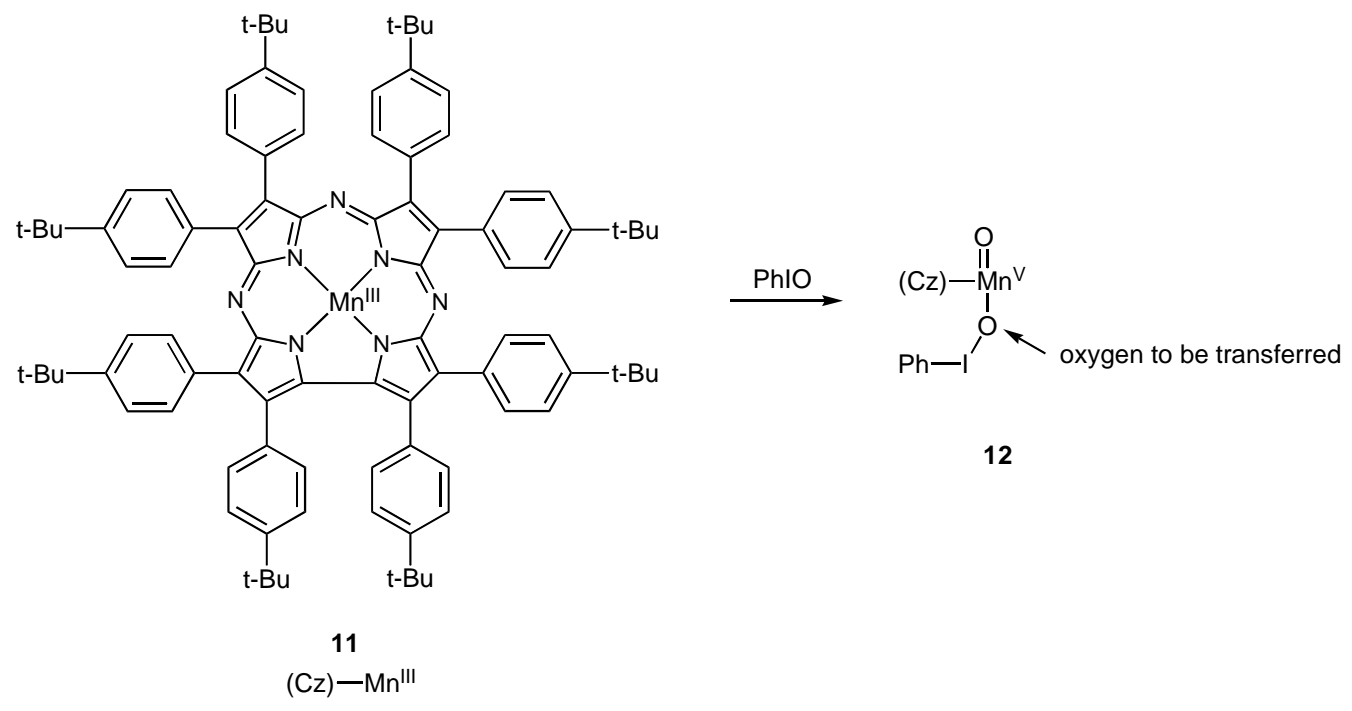

Metal-catalyzed epoxidations can also work quite efficiently even with very simple ligand systems. For example, 1-octene (13) is converted to its corresponding epoxide in practically quantitative yield within $5 \mathrm{~min}$ upon exposure to peracetic acid in the presence of the $\mathrm{Mn}$ (II)bipyridyl complex at $0.1 \mathrm{~mol} \%$ catalyst loading. ${ }^{9}$ 

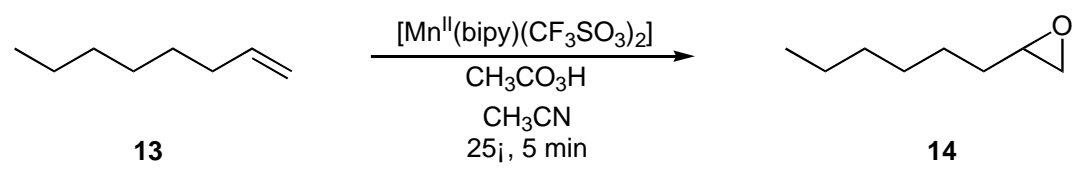

$99 \%$ conversion

$94 \%$ yield

Metal-catalyzed epoxidations are becoming important on the industrial scale, since the ability to use molecular oxygen as the terminal oxidant offers considerable operational and environmental benefits. The crucial feedstock propylene oxide (16) can be produced using molecular oxygen and a catalytic system of palladium(II) acetate and a peroxo-heteropoly compound in methanol. ${ }^{10}$ A discussion of some quantum chemical calculations with regard to the industrially relevant peroxometal epoxidation catalysts has recently appeared in the literature. ${ }^{11}$

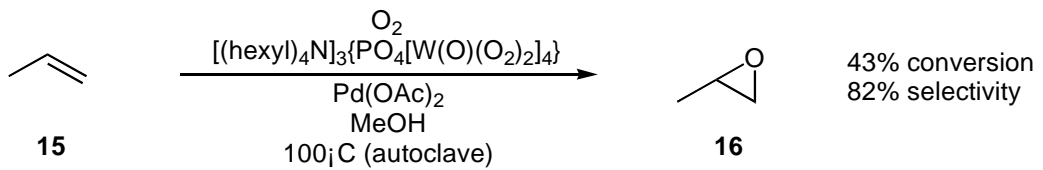

In the realm of epoxidations without the use of transition metals, dioxirane-mediated processes are among the most versatile. While the use of stoichiometric amounts of even the simplest dioxiranes can still be experimentally cumbersome, novel catalytic systems continue to emerge. For example, the PEG-immobilized trifluoroacetophenone $\mathbf{1 7}$ is a convenient dioxirane precursor that is highly active, soluble in both water and organic solvents, and easily recoverable and reusable. In the presence of Oxone, this ketone mediates the efficient epoxidation of sensitive substrates, such as the BOC-protected aminostyrene $18 .^{12}$
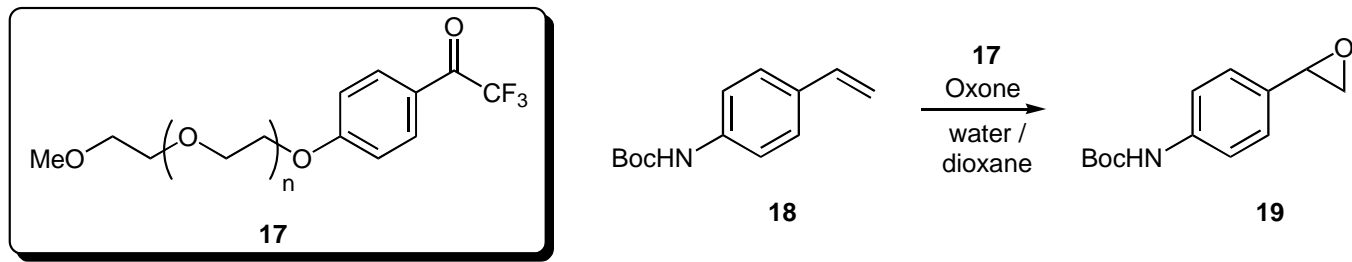

$90 \%$ yield

These ketone precursors can also serve as chiral auxiliaries. The dioxirane from the fructose-derived ketone 20 converts trisubstituted and trans-disubstitued alkenes (e.g., 22) to the corresponding epoxides in very good yields and enantioselectivities, but is less effective for terminal and cis-disubstituted substrates. Fortunately, the oxazolidino analog $\mathbf{2 1}$ exhibits a complementary scope, providing high enantioselectivities for these latter olefins (e.g., 24). The stereochemical outcome of the reaction has been rationalized on the basis of a spiro transition state. $^{13}$ Very mild homogeneous conditions have been elaborated for the in situ generation of dioxiranes from ketones using a buffered Oxone solution. ${ }^{14}$ 


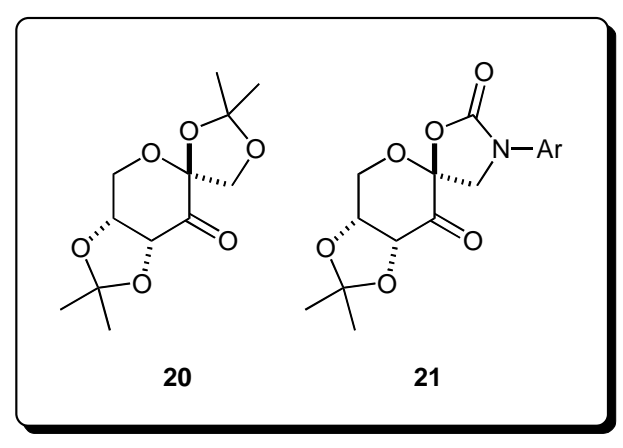

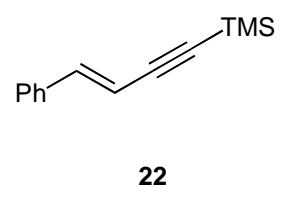
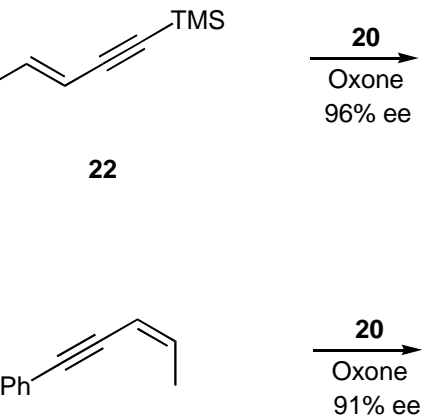

24

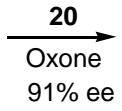

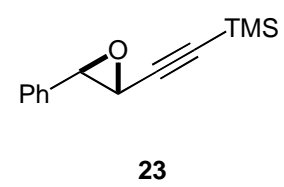

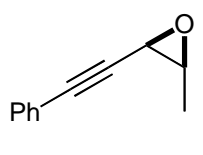

25

Page and co-workers have reported a noteworthy advance in asymmetric epoxidations of unfunctionalized olefins using chiral iminium salts, such as 26 . In the presence of Oxone neutralized with sodium carbonate, the iminium salt is converted to the corresponding oxaziridinium salt, which serves as the oxygen transfer agent. Yields are moderate to good, and enantioselectivities are highly variable, but can reach as high as $95 \%$, as exemplified by the epoxidation of 1-phenyl-3,4-dihydronaphthalene (27). Effective catalyst loadings can also be remarkably low, as shown in the conversion of 1-phenylcyclohexene (29), which proceeded in $91 \%$ ee with only $0.5 \mathrm{~mol} \%$ of the catalyst. 15 Anhydrous conditions have also been optimized for this system.

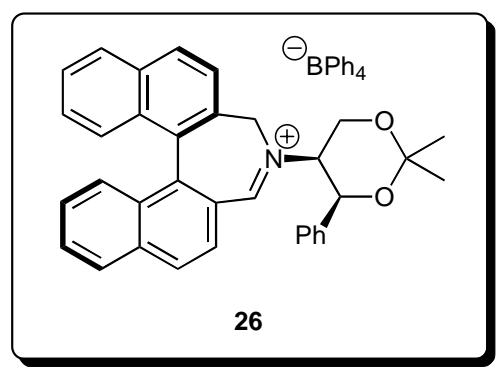

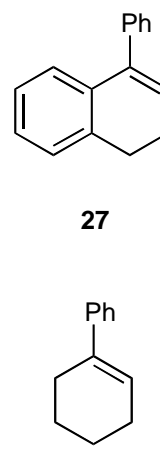

29
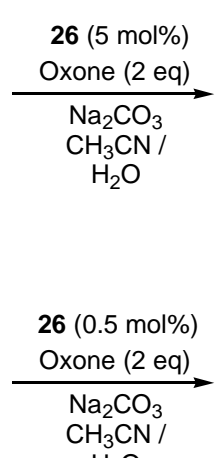

$\mathrm{H}_{2} \mathrm{O}$

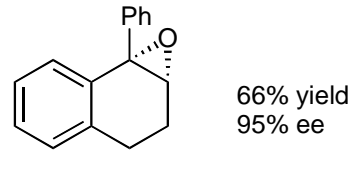

28

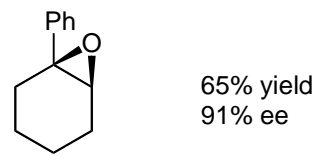

30

Remarkably, simple chiral ammonium salts such as 31 can induce modest enantiomeric excess in the Oxone-mediated epoxidation of trisubstituted alkenes. While no in-depth mechanistic models have been proposed, the mode of action is believed to be through the formation of chiral salts with Oxone itself. ${ }^{17}$ 

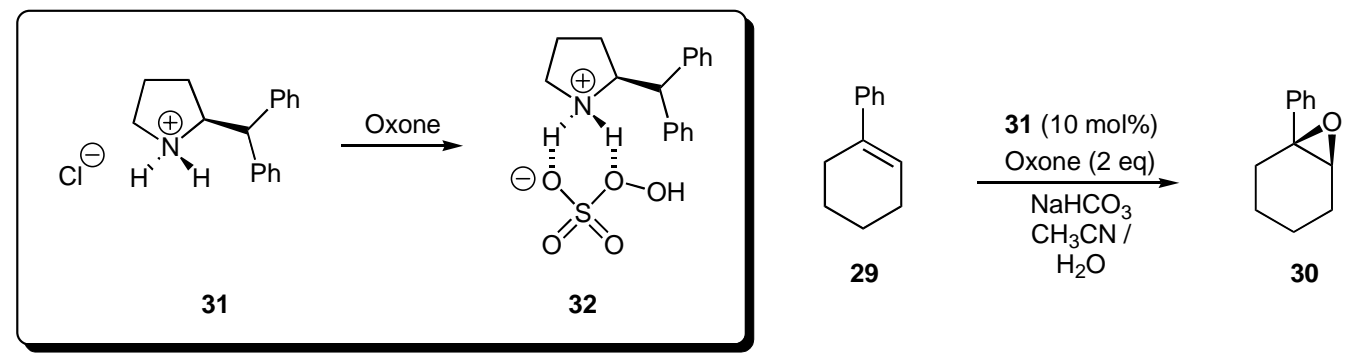

$93 \%$ yield

30

The same kind of associative event lies at the heart of the catalytic asymmetric epoxidation of enones using the interesting binaphthyl derived spiro ammonium salt 33, which serves as a phase transfer catalyst as well as chiral auxiliary. Using sodium hypochlorite in a biphasic system, this catalyst mediates the high-yielding epoxidation of a variety of electron-deficient trisubstituted and trans-disubstituted olefins with excellent enantioselectivity, as represented by the conversion of enone 34 to the corresponding epoxy ketone $35 .{ }^{18}$

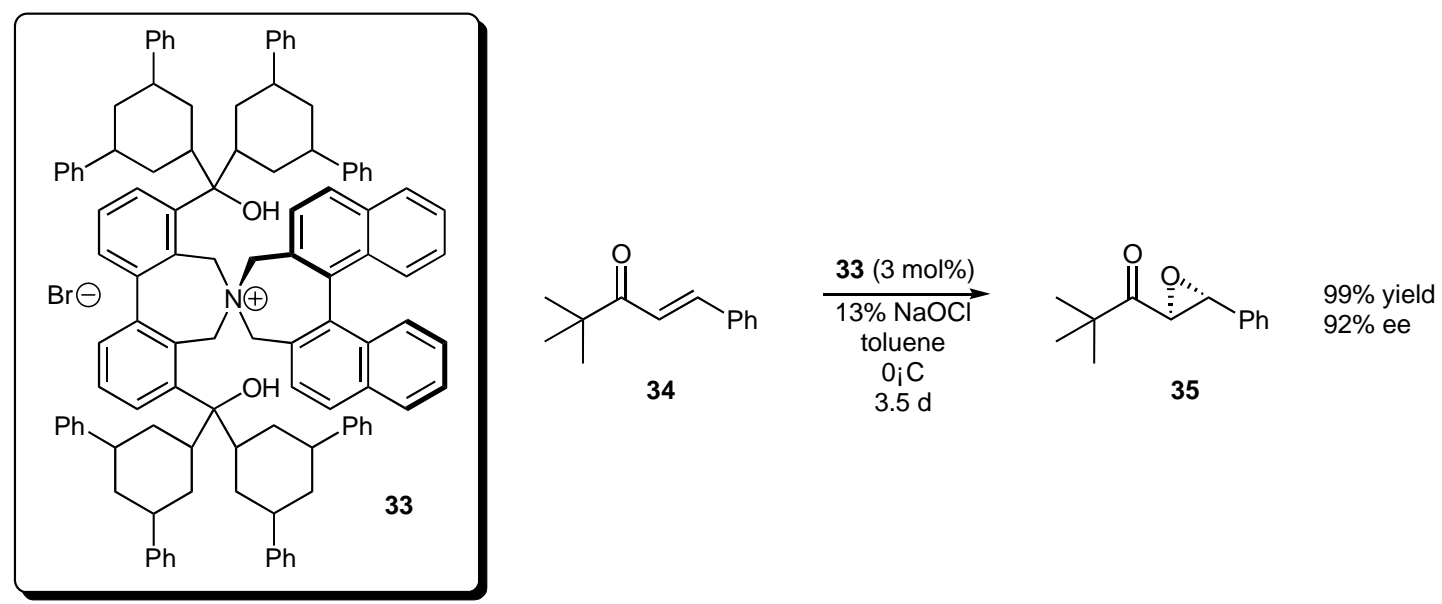

Another commonly employed approach for the enantioselective epoxidation of enones is the Juliá-Colonna procedure, which utilizes basic hydrogen peroxide as an oxygen source and polyleucine as a chiral auxiliary. Practical improvements to this protocol have recently been reported. In one modification, poly-(L)-leucine is stirred in a biphasic mixture of water/toluene with tetrabutylammonium bisulfate as a phase-transfer agent. The polyamino acid appears to be the species that consumes hydrogen peroxide from the aqueous medium and serves as a source of oxygen in the organic phase. These reaction conditions accomplished the notable feat of preparing the epoxysulfone 37 in high yield and good optical purity. ${ }^{19}$ Investigators at Bayer reported very similar conditions using silica supported poly-(L)-leucine, which was shown to epoxidize trans-chalcone (38) in excellent yield and enantiomeric excess. ${ }^{20}$ 

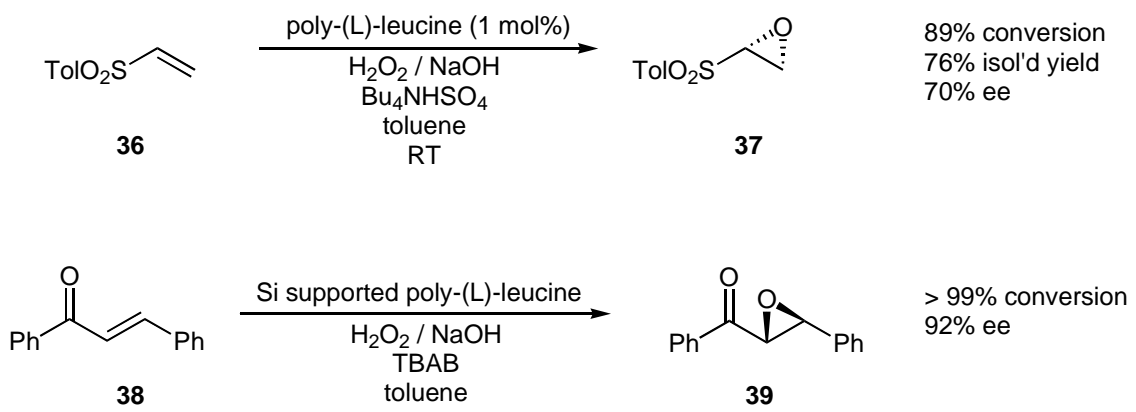

$\gamma$-Hydroxy- $\alpha, \beta$-unsaturated esters were shown to undergo diasteroselective epoxidation in the presence of lithium $t$-butylperoxide, in which the delivery of the epoxide oxygen is guided by the solvent system. Thus, in coordinating solvents such as THF, the predominant product (i.e. 41) is anti; conversely, non-polar solvents such as toluene lead to syn diastereoselectivity. Interestingly, temperature exerts practically no influence on the stereochemical outcome over the range of $-80^{\circ}-50^{\circ} \mathrm{C}^{21}$

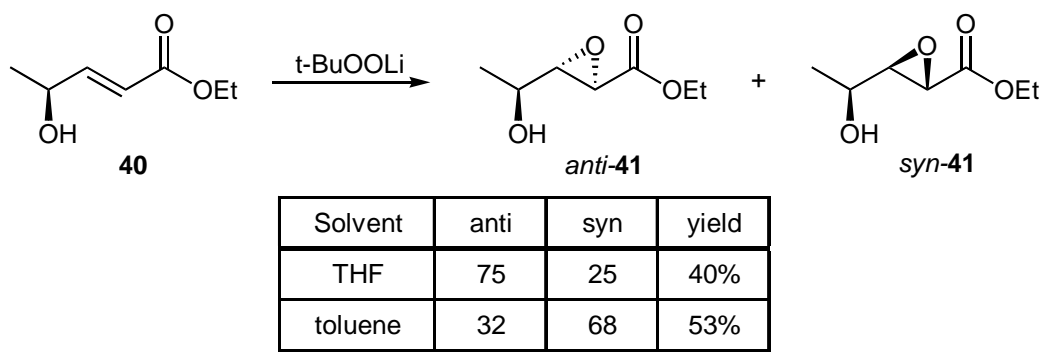

Thus far, discussion has centered around the reaction of alkenes with a source of electrophilic oxygen as a route to epoxides [the $\mathrm{C}=\mathrm{C}+\mathrm{O}$ protocol]. However, a second general approach is represented by the reaction of carbonyl compounds with amphophilic carbon centers [the $\mathrm{C}=\mathrm{O}+\mathrm{C}$ protocol]. For example, sulfonium ylides are known to convert aldehydes and ketones to epoxides; much recent work has focused on asymmetric induction using this methodology, a topic which has been the subject of a concise review in the past year. ${ }^{22}$ As an illustration, the D-mannitol derived chiral sulfide 42 serves as a useful chiral auxiliary in the sulfonium methylide epoxidation of aldehydes to provide terminal monosubstituted oxiranes $\left(\right.$ e.g., 44) in fair to excellent yield and good enantiomeric excess. ${ }^{23}$
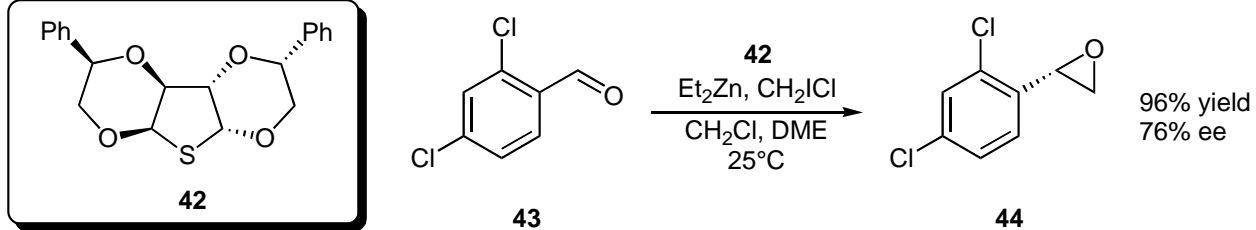

Aldehydes can also be converted to enantioenriched chiral epoxides through the Darzens reaction. Thus, haloamides (e.g., 47) react with benzaldehyde in the presence of a novel phase 
transfer catalyst (45) derived from BINOL to give 1,2-disubstituted epoxides in good yields with high enantioselectivity, and fair diastereoselectivity. The protocol can be applied to both aromatic and aliphatic aldehydes with low catalyst loadings. ${ }^{24}$

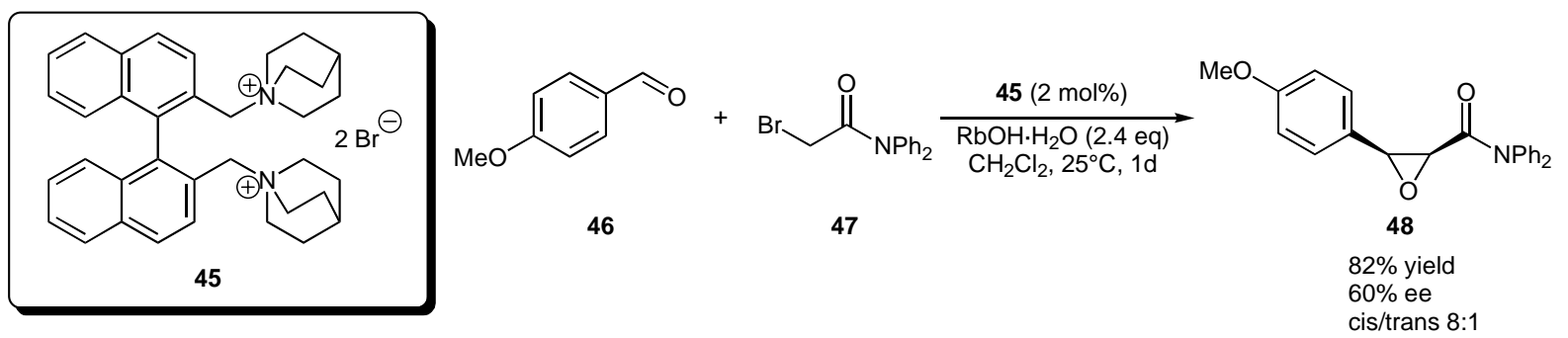

Finally, carbenoid species can be used as the carbon donor in aldehyde epoxidations. Thus, the rhodium carbenoid derived from the cyclic diazoamide $\mathbf{4 9}$ and rhodium(II) acetate reacts stereoselectively with aryl aldehydes to provide spiro-indolooxiranes $\mathbf{5 0}$ with Zstereochemistry. The reaction is believed to proceed via the formation of a carbonyl ylide (51), which undergoes stereospecific thermal conrotatory electrocyclization to form the observed epoxide. $^{25}$

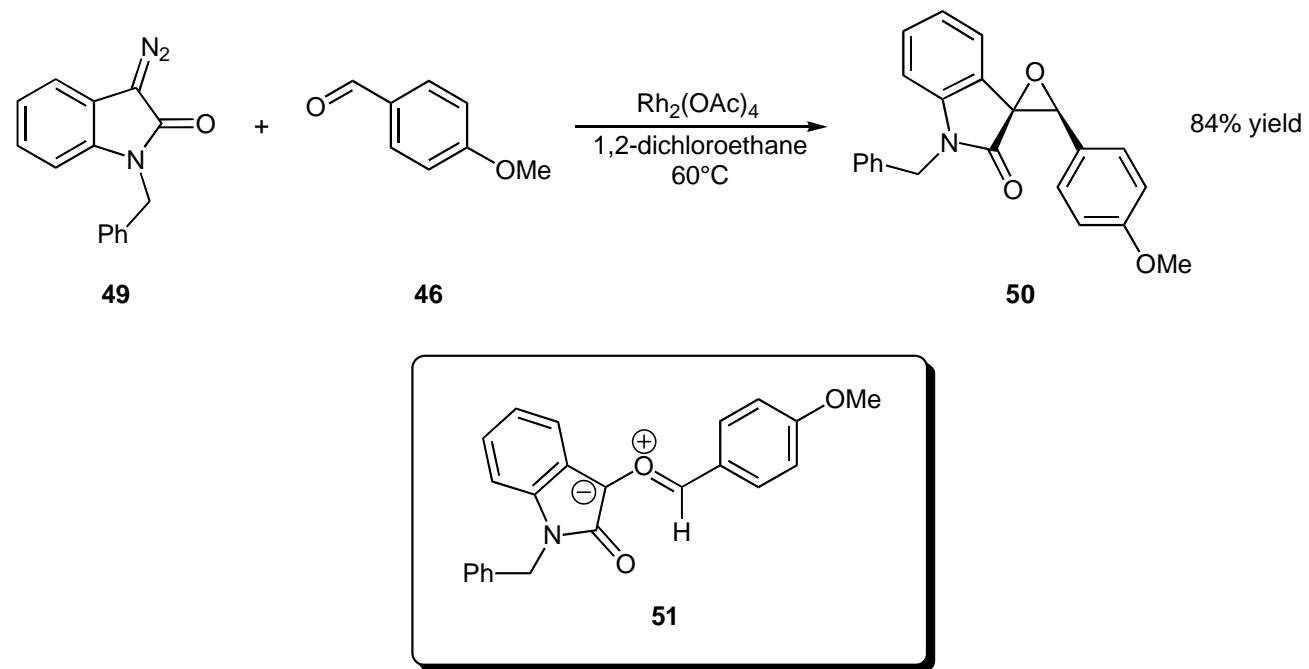

\section{Reactions of Epoxides}

The hydrolysis of epoxides is a well-known reaction which can be exploited for various synthetically useful outcomes. Chiral nonracemic epoxides can be prepared from their racemates through the salen-mediated hydrolytic kinetic resolution (HKR). Racemic epichlorohydrin (53) was resolved in the presence of catalyst 52 and a slight excess of water under solvent-free conditions. The catalyst counterion exerts a significant effect on the course of the reaction, presumably due to competitive addition onto the epoxide, an effect which is evident in apparent 
reaction rates, but not enantioselectivities. Less nucleophilic counterions, such as tosylate, lead to more rapid resolution and lower catalyst loading requirements. ${ }^{26}$
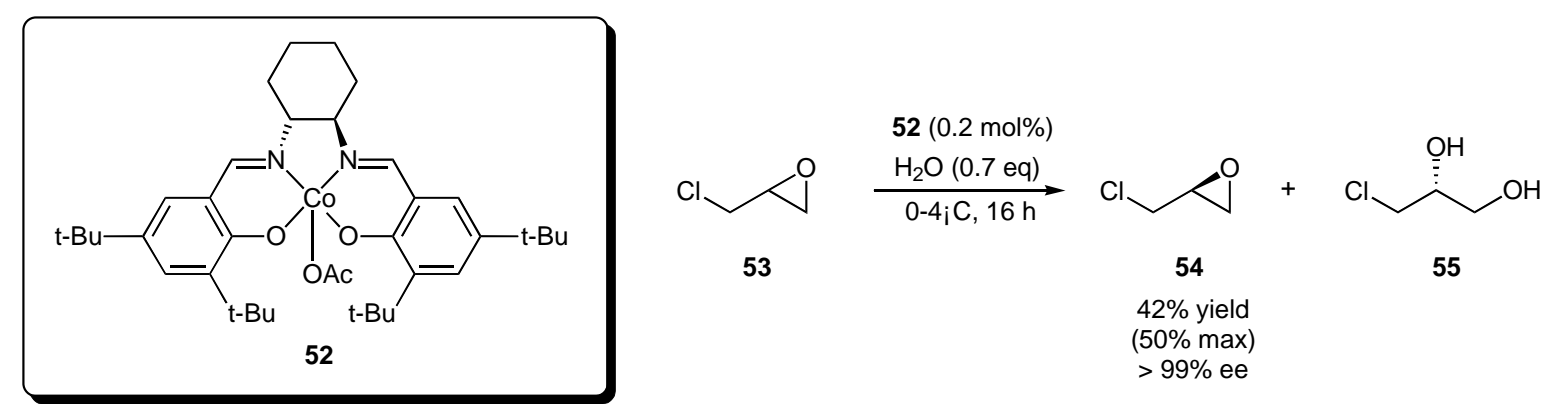

Chiral diols have been prepared from the desymmetrization of the corresponding meso epoxides using epoxide hydrolases (EHs). As a general trend, microbial EHs tend to prefer the generation of $(R, R)$-diols, as demonstrated by the desymmetrization of cis-stilbene oxide (56). However, a recently reported variant (BD9126) exhibits the opposite enantioselectivity. This nice complement to existing methods provides good to excellent ee's, albeit at reaction rates typically two orders of magnitude less than the $(R, R)$-producing enzymes. ${ }^{27}$ A new palladiumcatalyzed ring opening of epoxyacrylate derivatives mediated by boric acid gives the corresponding diols (59) with net retention of configuration at both epoxide carbon centers, providing a nice complement to osmium dihydroxylation methodology. The reaction is believed to proceed through the intermediacy of cyclic boronates $($ e.g., 60$){ }^{28}$
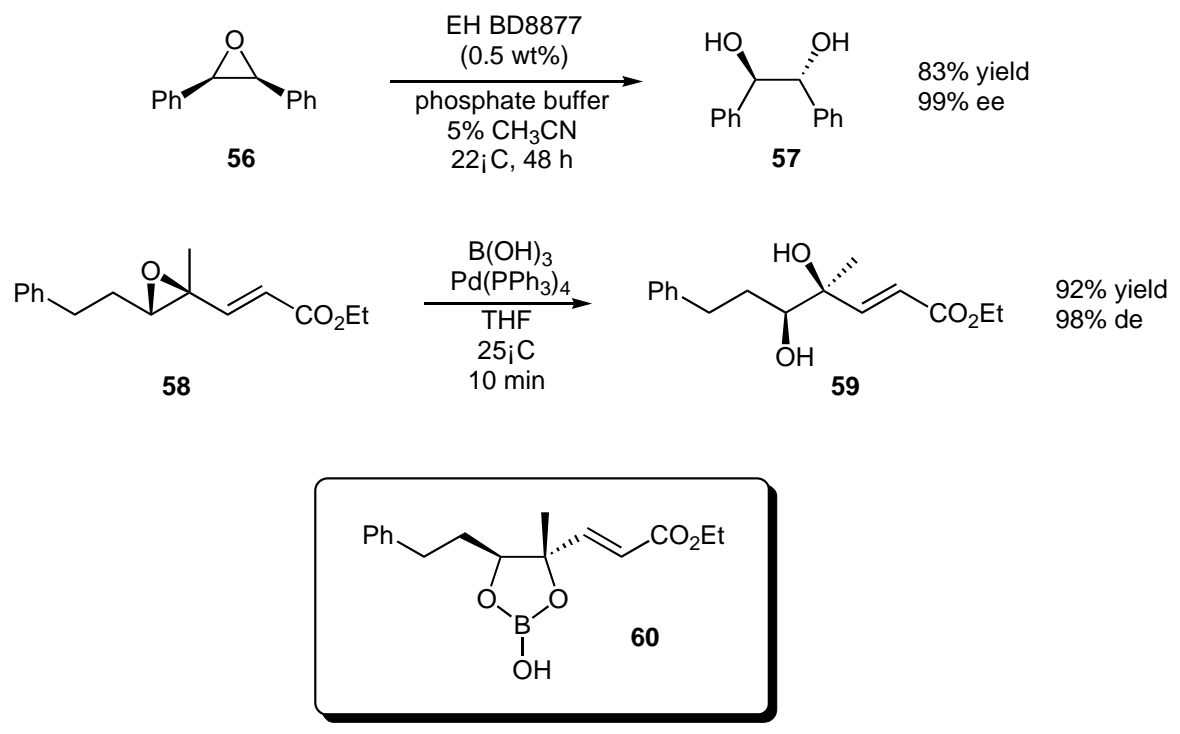

Epoxides also suffer nucleophilic attack by amines, forming ethanolamine derivatives. This reaction can take place under a variety of conditions and with the influence of several catalysts. Styrene oxide (61) undergoes ring opening in the absence of catalyst under strictly 
thermal conditions $\left(90^{\circ} \mathrm{C}\right.$ in a sealed tube) to give predominantly the amino-alcohol 62 , the product of attack at the more substituted $(\alpha)$ position. ${ }^{29}$ The reaction time can be reduced by using a combination of catalytic bismuth(III) trifluoroacetate and brief microwave irradiation, as illustrated in the reaction of $\mathbf{6 1}$ with $p$-bromoaniline, which requires only $40 \mathrm{sec}$ of heating in a conventional microwave to provide 62 as the exclusive product in $90 \%$ yield. $^{30}$ The reaction temperature can also be lowered (to room temperature) by the addition of $5 \mathrm{~mol} \%$ lithium bromide as a catalyst. Under these conditions, the regioselectivity appears to be dependent upon the attacking nucleophile. Thus, $p$-chloroaniline engages in exclusive attack at the $\alpha$-position, whereas piperidine gives an almost equimolar mixture of products 62 and $63 .^{31}$ In contrast, the use of nickel(II) acetate as catalyst leads to an extremely high regioselectivity with piperidine, favoring the $\alpha$-product (62). ${ }^{32}$ Interestingly, indium bromide promotes the opposite regiochemistry. Thus, treatment of styrene oxide with $p$-tert-butylaniline and 1 equiv of indium tribromide gives exclusively aminoalcohol 63 in 75\% yield. In this protocol, attempts to use substoichiometric amounts of catalyst led to lower yields and significant amounts of a 2:1 adduct of epoxide to amine.

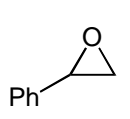

61

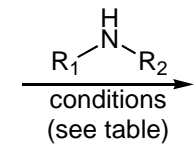

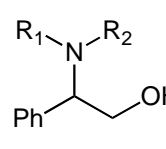

62

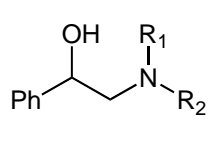

63

\begin{tabular}{|c|c|c|c|c|c|c|c|c|c|}
\hline amine & $\mathrm{R}_{1}$ & $\mathrm{R}_{2}$ & catalyst (loading) & solvent & time & temp & yield & $\mathbf{6 2 : 6 3}$ & reference \\
\hline ethanolamine & $\mathrm{HOCH}_{2} \mathrm{CH}_{2}{ }^{-}$ & $\mathrm{H}-$ & none & neat & $3 \mathrm{~h}$ & $90^{\circ} \mathrm{C}^{*}$ & $99 \%$ & $90: 10$ & $<04 \mathrm{SC} 2393>$ \\
\hline p-bromoaniline & $(\mathrm{p}-\mathrm{Br}) \mathrm{Ph}-$ & $\mathrm{H}-$ & $\mathrm{Bi}(\mathrm{TFA})_{3}$ & $\mathrm{CH}_{3} \mathrm{CN}$ & $40 \mathrm{~s}$ & $\mathrm{MW}$ & $90 \%$ & $100: 0$ & $<04 \mathrm{CL} 304>$ \\
\hline p-chloroaniline & $(\mathrm{p}$-Cl)Ph- & $\mathrm{H}-$ & $\mathrm{LiBr}(5 \mathrm{~mol} \%)$ & neat & $5 \mathrm{~h}$ & $\mathrm{RT}$ & $100 \%$ & $100: 0$ & $<04 \mathrm{EJOC} 3597>$ \\
\hline piperidine & \multicolumn{2}{|c|}{$-\left(\mathrm{CH}_{2}\right)_{5^{-}}$} & $\mathrm{LiBr}(5 \mathrm{~mol} \%)$ & neat & $5 \mathrm{~h}$ & $\mathrm{RT}$ & $98 \%$ & $42: 58$ & $<04 \mathrm{EJOC} 3597>$ \\
\hline piperidine & \multicolumn{2}{|c|}{$-\left(\mathrm{CH}_{2}\right)_{5^{-}}$} & $\mathrm{Ni}(\mathrm{OAC})_{2} \cdot 2 \mathrm{H}_{2} \mathrm{O}$ & neat & $12 \mathrm{~h}$ & $50^{\circ} \mathrm{C}$ & $99 \%$ & $96: 4$ & $<04 \mathrm{SL} 846>$ \\
\hline p-t-butylaniline & $(\mathrm{p}$-tBu $) \mathrm{Ph}-$ & $\mathrm{H}-$ & $\mathrm{InBr}_{3}(1 \mathrm{eq})$ & $\mathrm{CH}_{2} \mathrm{Cl}_{2}$ & $12 \mathrm{~h}$ & $\mathrm{RT}$ & $75 \%$ & $0: 100$ & $<04 \mathrm{TL} 7495>$
\end{tabular}

*sealed tube

Similar conditions have been applied to the ring-opening of meso epoxides using amines. Bismuth triflate catalyzes the reaction of cyclohexene oxide (64) with $p$-bromoaniline under aqueous conditions to provide the $\beta$-aminoalcohol 65 in $84 \%$ yield. In this particular case, the water solubility of the starting materials required the use of a micellar solution of sodium dodecyl sulfate (SDS); however, more soluble amines could be employed in water and bismuth triflate alone. $^{34}$ A lanthanide variant has also been reported. Thus, treatment of 64 with $p$ bromoaniline and $10 \%$ samarium iodide in methylene chloride solution provides the product 65 in $76 \%$ yield. An interesting aspect of the latter protocol is that the chiral bis-binaphthoxy iodo samarium catalyst (66) has been shown to effectively desymmetrize meso epoxides, as illustrated by the conversion of the cyclohexadiene oxide 67 to the nonracemic aminoalcohol $68 .{ }^{35}$ 


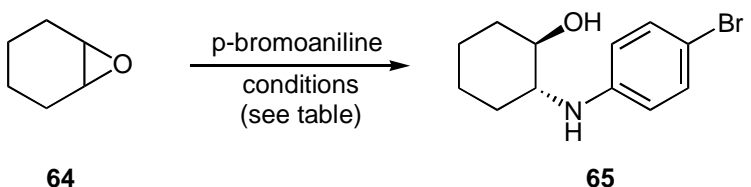

64

65

\begin{tabular}{|c|c|c|c|c|}
\hline conditions & time & temp & yield & reference \\
\hline $\mathrm{Bi}(\mathrm{OTf})_{3}(10 \mathrm{~mol} \%), \mathrm{SDS}(40 \%), \mathrm{H}_{2} \mathrm{O}$ & $9 \mathrm{~h}$ & $25^{\circ} \mathrm{C}$ & $84 \%$ & $<04 \mathrm{TL} 49>$ \\
\hline $\mathrm{Sml}_{2}(\mathrm{THF})_{2}(10 \mathrm{~mol} \%), \mathrm{CH}_{2} \mathrm{Cl}_{2}$ & $18 \mathrm{~h}$ & $25^{\circ} \mathrm{C}$ & $76 \%$ & $<04 \mathrm{TL} 7749>$ \\
\hline
\end{tabular}
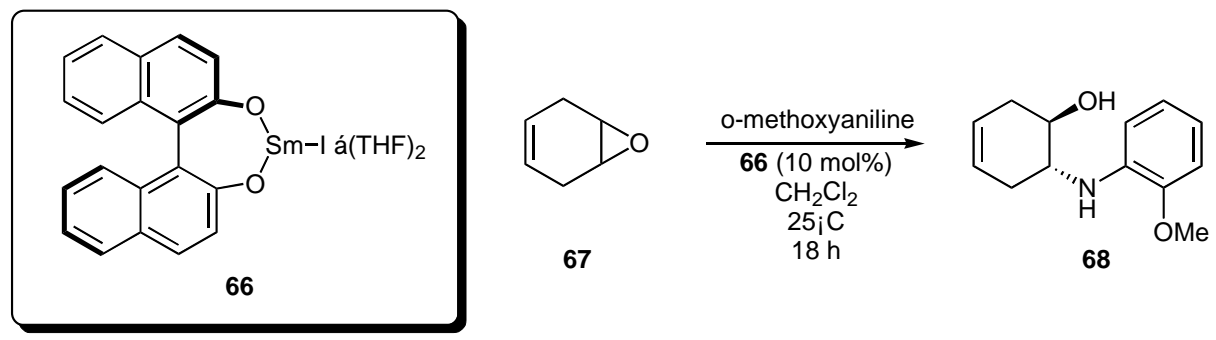

$100 \%$ conversion

$60 \%$ yield

$68 \%$ ee

Chromium(salen) catalysts (e.g., 69) are also useful in desymmetrizing meso epoxides. Thus, cis-stilbene oxide (70) is converted to the $(S, S)$-aminoalcohol 71 in the presence of catalytic quantities of $\mathbf{6 9}$ in methylene chloride solution open to the atmosphere. The addition of small quantities of triethylamine was found to dramatically increase enantioselectivities (by almost 25\%). This catalytic system also promotes an efficient aminolytic kinetic resolution (AKR) of racemic epoxides with C2-type symmetry. ${ }^{36}$
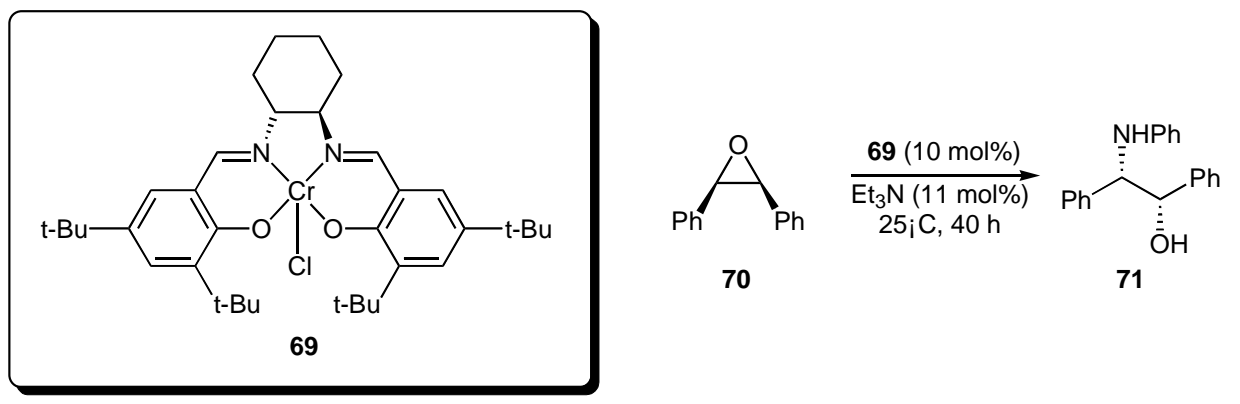

$98 \%$ yield

70

71

Another very useful method of introducing nitrogen during epoxide cleavage is based on the azide nucleophile. This approach was used in the regioselective ring-opening of the optically pure aryl epoxide 72, a key step in the synthesis of (-)-cytoxazone (74), a cytokine modulating natural product isolated from Streptomyces. The regioselectivity is substrate dependent: terminal epoxides are attacked preferentially at the less substituted position, whereas benzylic positions tend to be more electrophilic in the case of aryl epoxides. The regioselectivity is attributed to the potassium ions found in mesoporous $4 \AA$ molecular sieves used as a catalyst. ${ }^{37}$ 


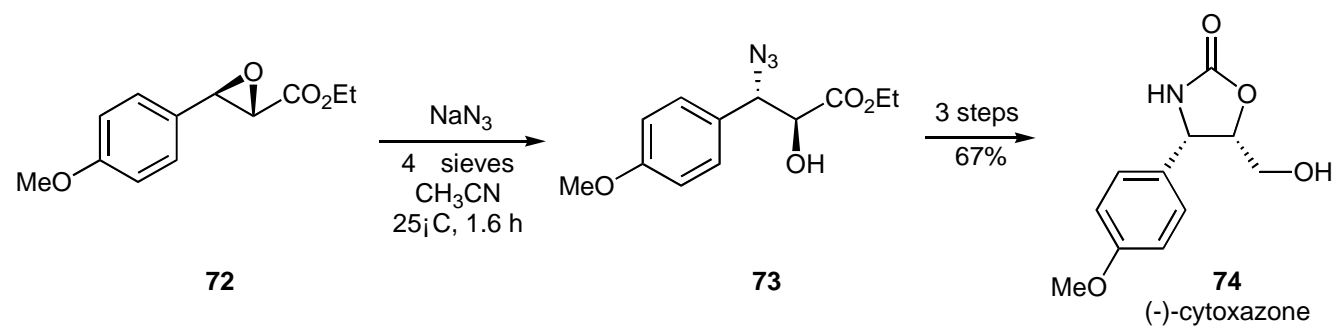

This type of ring-opening by heteroatomic nucleophiles can also occur in an intramolecular fashion, leading to new heterocycles. For example, vinyl epoxide 75 undergoes 6-endo cyclization in the presence of a rhodium catalyst to give the trans-hydroxypiperidine derivative 76 under extremely mild conditions. The olefinic moiety is crucial, as the reaction proceeds through the initial coordination of the $\pi$-bond and nitrogen lone pair, followed by the formation of an enyl or $\pi$-allyl intermediate. This double coordination is believed to govern both the regioselectivity and stereoselectivity of the process. ${ }^{38}$

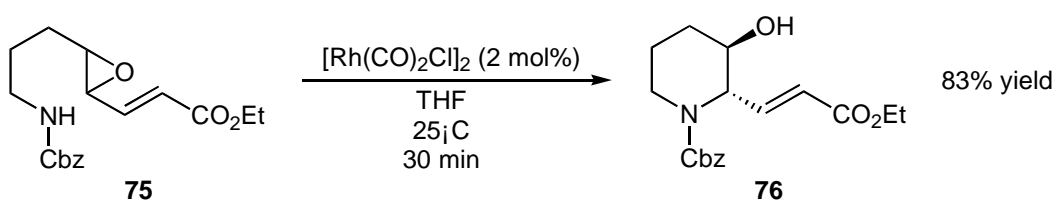

Epoxides can serve as competent electrophiles in the alkylation of a variety of carbanions, as illustrated by the ring opening of cyclohexene oxide (64) with the dianion of phenylacetic acid (77) to produce the $\gamma$-hydroxy carboxylic acid 78. In this protocol, the dianion is generated using $n$-butyllithium and a substoichiometric quantity of a secondary amine; lithium chloride is also used as a Lewis acid additive to activate the secondary epoxide toward nucleophilic addition. Primary epoxides undergo addition without the use of catalyst - in these cases, the nucleophile attacks at the less substituted position. ${ }^{39}$

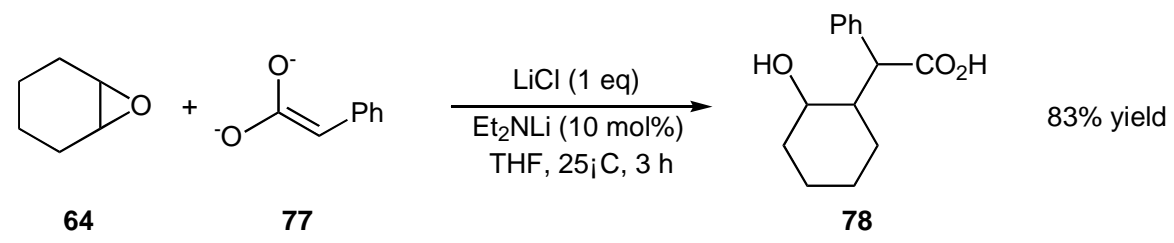

A similar transformation can be carried out under milder conditions by taking advantage of silyl ketene acetals, a masked form of the carboxylate dianion. When epichlorohydrin (53) was treated with the ketene acetal $\mathbf{7 9}$ in the presence of titanium(IV) chloride, a regioselective epoxide ring opening occurs at the less substituted carbon. Treatment of the crude reaction mixture with catalytic $p$-toluenesulfonic acid promoted a lactonization to the $\gamma$-butanolide $\mathbf{8 0}$ in high overall yield. ${ }^{40}$ 


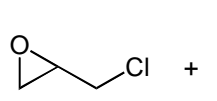

53

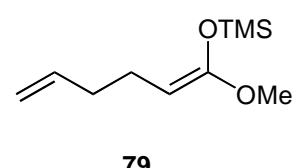

79

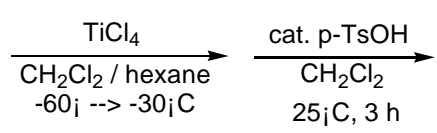

$25 i \mathrm{C}, 3 \mathrm{~h}$

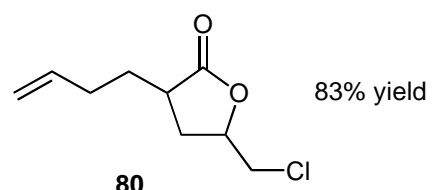

80

Other carbon nucleophiles also tend to attack at the less substituted position of an unsymmetrical epoxide. This holds true for cyanide, which can be conveniently prepared in situ from methyllithium and acetone cyanohydrin (82). In a one-pot reaction, the epoxy ether $\mathbf{8 1}$ is converted to hydroxy nitrile 83 in $80 \%$ yield. ${ }^{41}$ Similarly, alkyllithium reagents attack the unsubstituted carbon of terminal epoxides, and when an excess of base is present, the intermediate alkoxides undergo elimination to form alkenes. Thus, when decyl epoxide (84) is treated with the vinyl lithium derivative 85 in the presence of lithium tetramethylpiperidine (LTMP), diene $\mathbf{8 8}$ is produced exclusively in the E-geometry in very good yield. The mechanism involves an initial deprotonation of the epoxide by LTMP, followed by nucleophilic attack of the carbenoid oxiranyl anion by the vinyllithium 85. The dilithio species 87 suffers elimination to provide the observed alkene. ${ }^{42}$

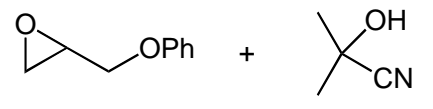

81

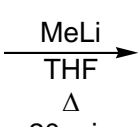

$20 \min$

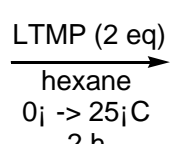

$2 \mathrm{~h}$

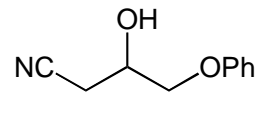

83

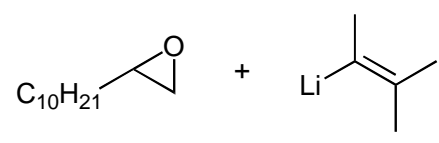

84

85

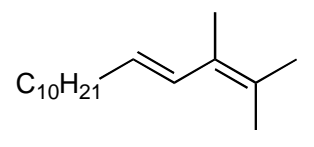

88
$80 \%$ yield

$85 \%$ yield

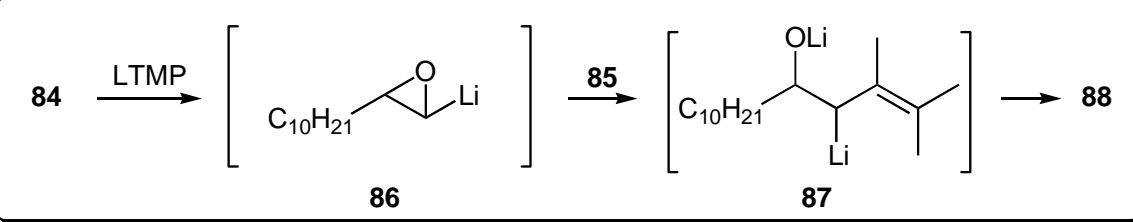

The fascinating carbenoid character of the epoxide anion is also manifested in an intramolecular cyclopropanation reaction, in which the anion adds across a tethered olefin to provide bicyclo[3.1.0]hexanols (e.g., 92). The reaction is remarkably chemo- and diastereoselective. No $\mathrm{C}-\mathrm{H}$ insertion is observed, and yields are generally very good. The stereochemical outcome is rationalized on the basis of a trans-lithiation, as well as the geometric constraints imposed by the [3.1.0] bicyclic system. 43 In the absence of well-positioned double bonds, the oxiranyl anions can undergo another well-known reaction of carbenes, namely a 1,2hydride shift. In the case of these substrates, the rearrangement leads to the formation of an enolate anion. Treatment of the cyclohexyl alkynyl epoxide 93 with an excess of $n$-butyllithium leads to an oxiranyl anion at the allylic site (i.e., 94). This intermediate undergoes a formal 1,2- 
hydride shift to provide the enynolate 96, which undergoes double distal protonation during acidic work-up to form the allenone 98 in $64 \%$ yield. $^{44}$

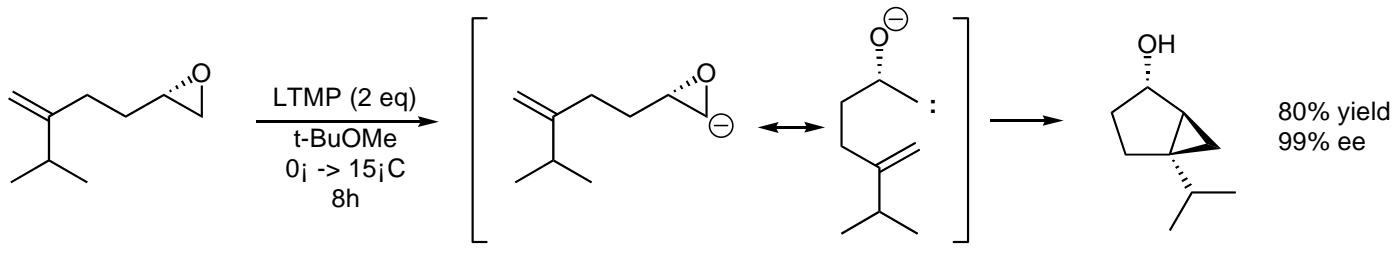

(S)-89

90

91

$(1 S, 2 S, 5 R)-92$
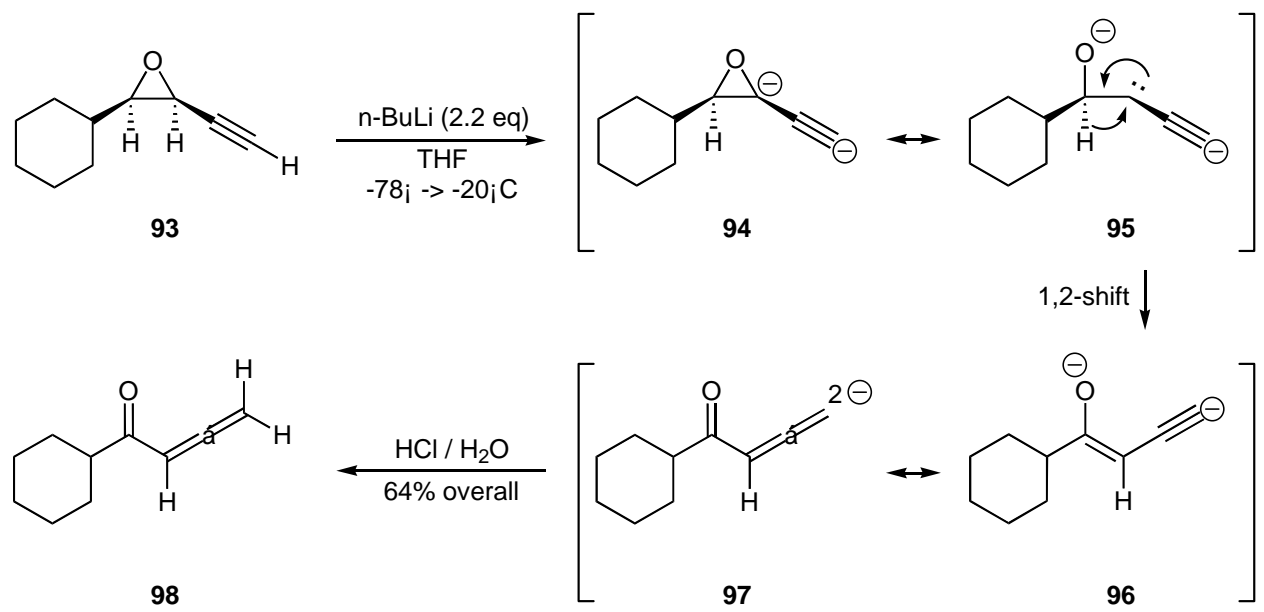

Lithiated epoxides are more commonly trapped by electrophiles, generating elaborated oxiranes. In this arena, Hodgson and co-workers ${ }^{45}$ have optimized the lithiation of nonstabilized terminal epoxides with sec-butyllithium assisted by diamine ligands, such as dibutylbispidine (DBB, 99) or (-)-sparteine (100). The oxiranyl anions thus formed engage in smooth nucleophilic addition onto aldehydes to form epoxyalcohols (e.g., 101); the same conditions can be used for the stannylation of epoxides (e.g., 84 -> 102). Similarly, epoxydisilanes (103) can be accessed through two sequences of deprotonation followed by treatment with chlorotrimethylsilane.
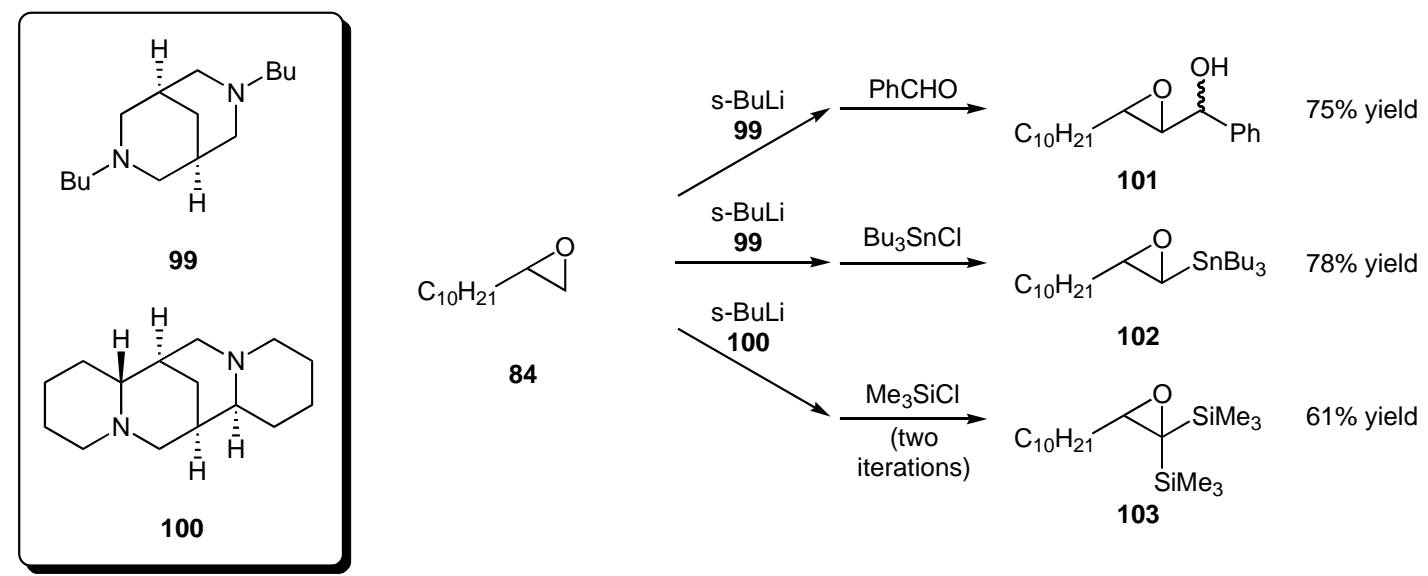
Epoxides undergo a variety of interesting and synthetically useful rearrangements to provide carbonyl compounds. The siloxycyclopentyl epoxide 104 (derived from the DMD oxidation of the corresponding tetrahydropyridine) underwent Lewis acid-catalyzed ring expansion to provide spiroketone $\mathbf{1 0 5}$ as a single diastereomer, suggesting a synchronous reaction mechanism. ${ }^{47}$ In a similar vein, the dichlororuthenium(IV)-porphyrin catalyst 106 promotes the rearrangement of terminal epoxides (e.g., 108) to aldehydes (e.g., 109). The epoxides themselves are prepared by oxidation of terminal alkenes (e.g., 107) with 106 in the presence of the stoichiometric oxidant 2,6-dichloropyridine $\mathrm{N}$-oxide $\left(\mathrm{Cl}_{2} \mathrm{pyNO}\right)$, thereby representing a novel conversion of terminal alkenes to aldehydes. ${ }^{48}$ Ruthenium catalysts also promote other unusual cyclizations of epoxides, such as the rearrangement of enynyl epoxide $\mathbf{1 1 0}$ to the cyclohexa-2,4-dienone 111, ${ }^{4} 9$ and the cyclization of $o$-ethynylphenyl epoxide $\mathbf{1 1 2}$ to the alkylidene indanone $113 .^{50}$ Both processes are thought to proceed through a rutheniumketene intermediate.
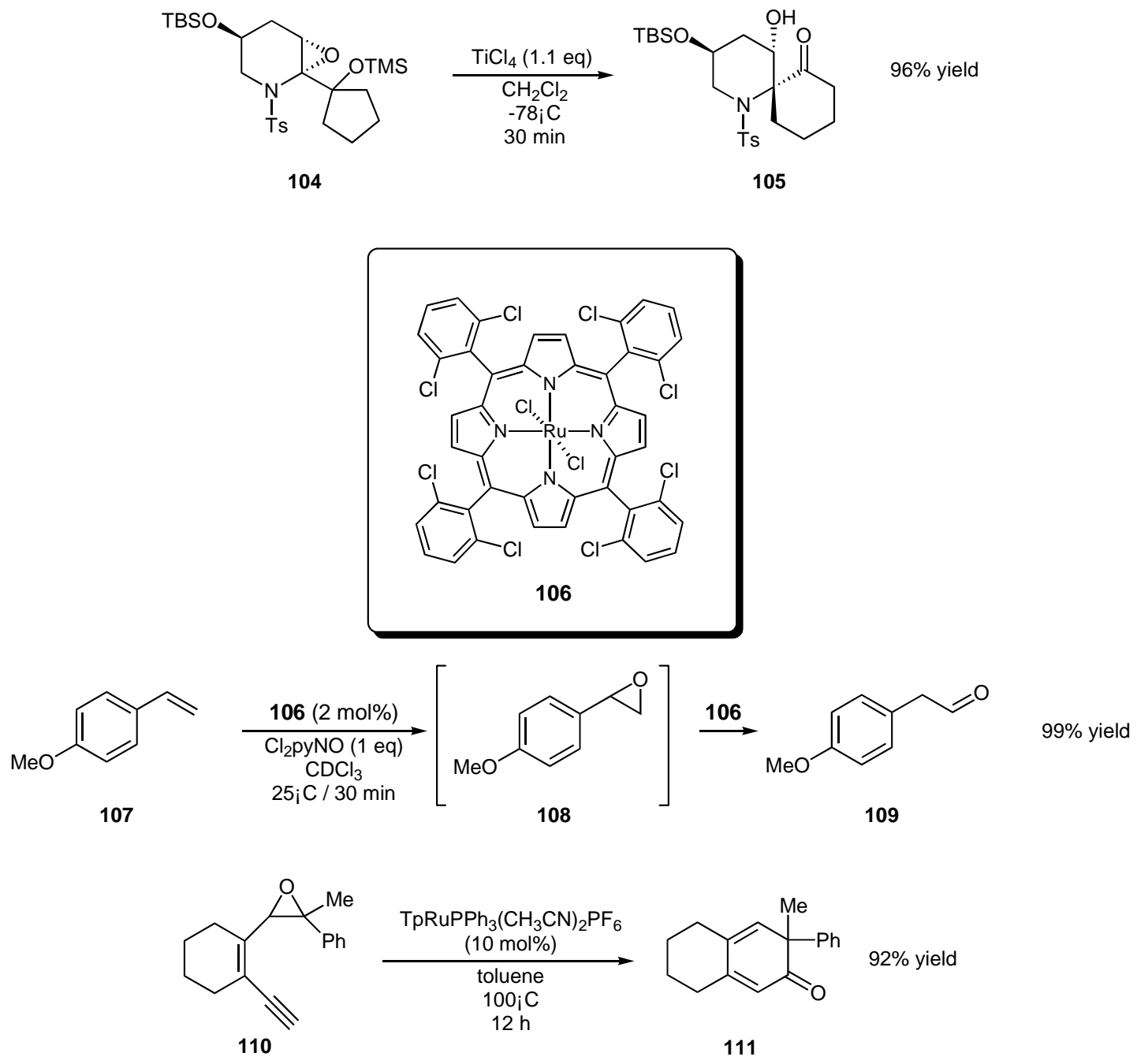


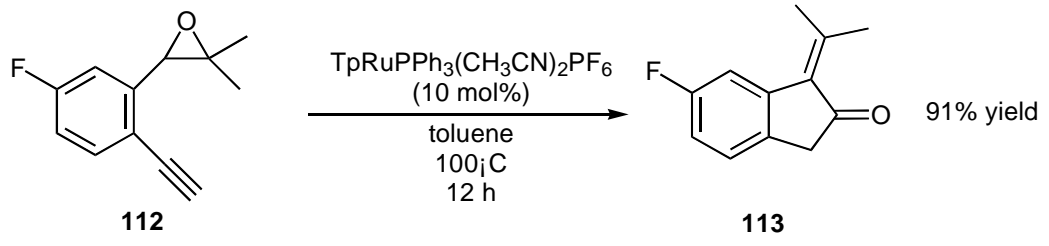

\section{Aziridines}

\section{Preparation of Aziridines}

One method widely used for the synthesis of aziridines is the addition of nitrenes to olefins. Along these lines, sulfonamides serve as convenient nitrene precursors, which can be converted using commercially available iodobenzene diacetate in the presence of various transition-metal catalysts. A copper(I) catalyst derived from the Evans chiral bis(oxazoline) ligand (114) was found to promote the high-yielding aziridination of styrene (4) with good enantioselectivity. ${ }^{51}$ Chang and co-workers have developed a copper-catalyzed variant that requires no external ligand. Instead, a pyridyl nitrogen onboard the sulfonamide moiety serves as an internal ligand (i.e., 118), which in turn increases the efficiency of the aziridination. ${ }^{52}$ Several unsaturated sulfonamides (e.g., 120) were shown to undergo a very facile intramolecular aziridination in the presence of a rhodium catalyst to provide tricyclic aziridines $(e . g ., 121) .^{53}$
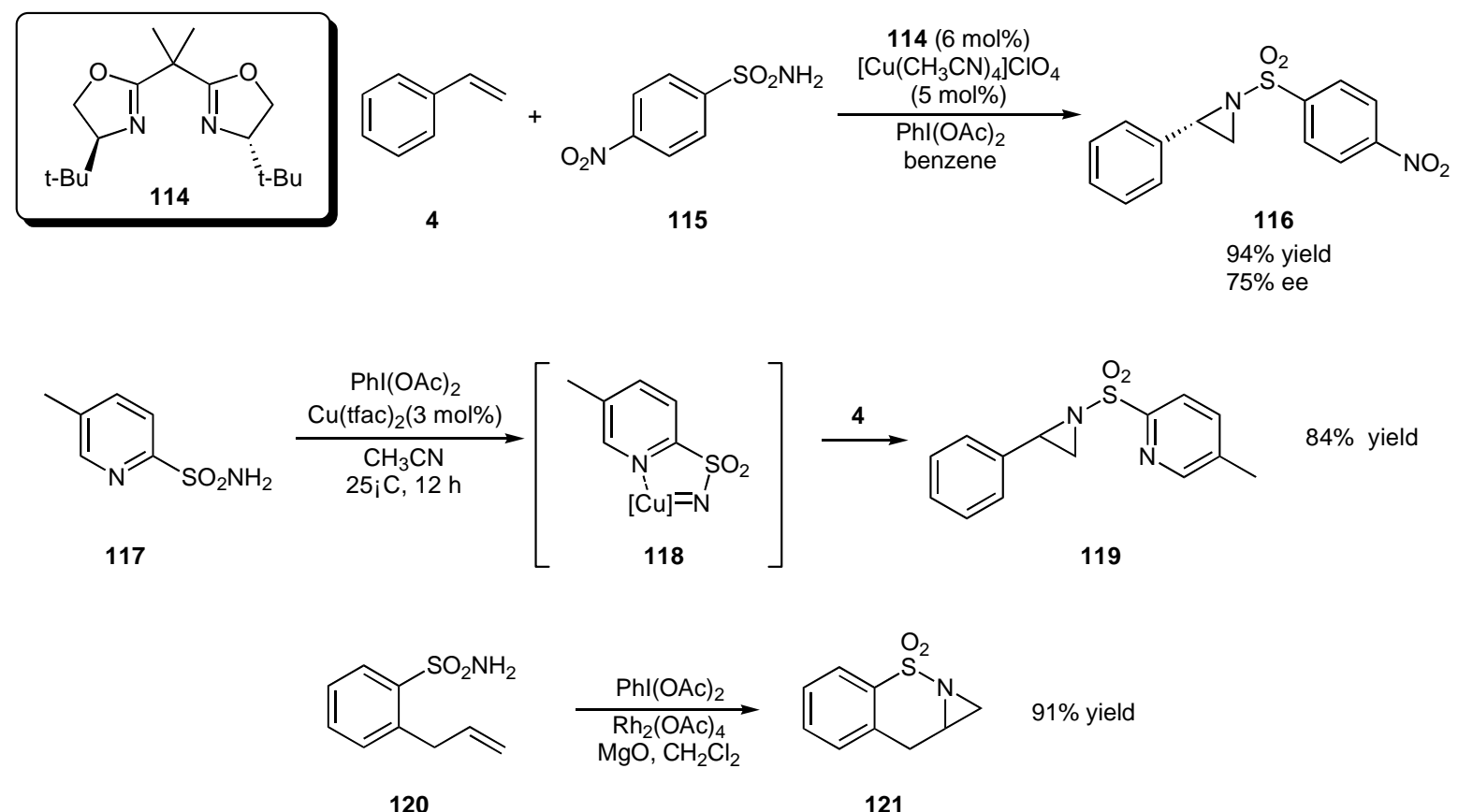

Other interesting catalyst systems include copper(II) acetylacetonate (acac) immobilized in ionic liquids such as 1-n-butyl-3-methylimidazolium tetrafluoroborate (bmimBF 4$)$, which facilitates catalyst recycling and also appears to accelerate the reaction. Thus, trans- 
methylstyrene (122) was converted to the corresponding tosyl aziridine (123) within 10 min using (tosylimino)phenyliodinane as the nitrogen donor. ${ }^{54}$ Bromamine $\mathrm{T}$ (125) is also a convenient, stable, and commercially available nitrene precursor. The perfluoroaryl iron porphyrin catalyst $\mathbf{1 2 4}$ is effective in promoting aziridination of a wide spectrum of alkenes in fair to good yields, as illustrated by the conversion of styrene (4) to the corresponding $N$-tosyl aziridine (126), which is believed to proceed through a mechanism involving an iron-nitrene intermediate. ${ }^{55}$ A fluorinated aryl ligand is also at the heart of a chiral ruthenium(salen) catalyst (127) designed for the purpose of effecting enantioselective aziridination of olefins using sulfonyl azides as nitrene precursors. Thus, $p$-bromostyrene (128) provides the corresponding aziridine (129) in the presence of tosyl azide and catalyst 127. Enantioselectivities can reach as high as $99 \%$, and the protocol also works well when tosyl azide is substituted with nosyl azide. ${ }^{56}$
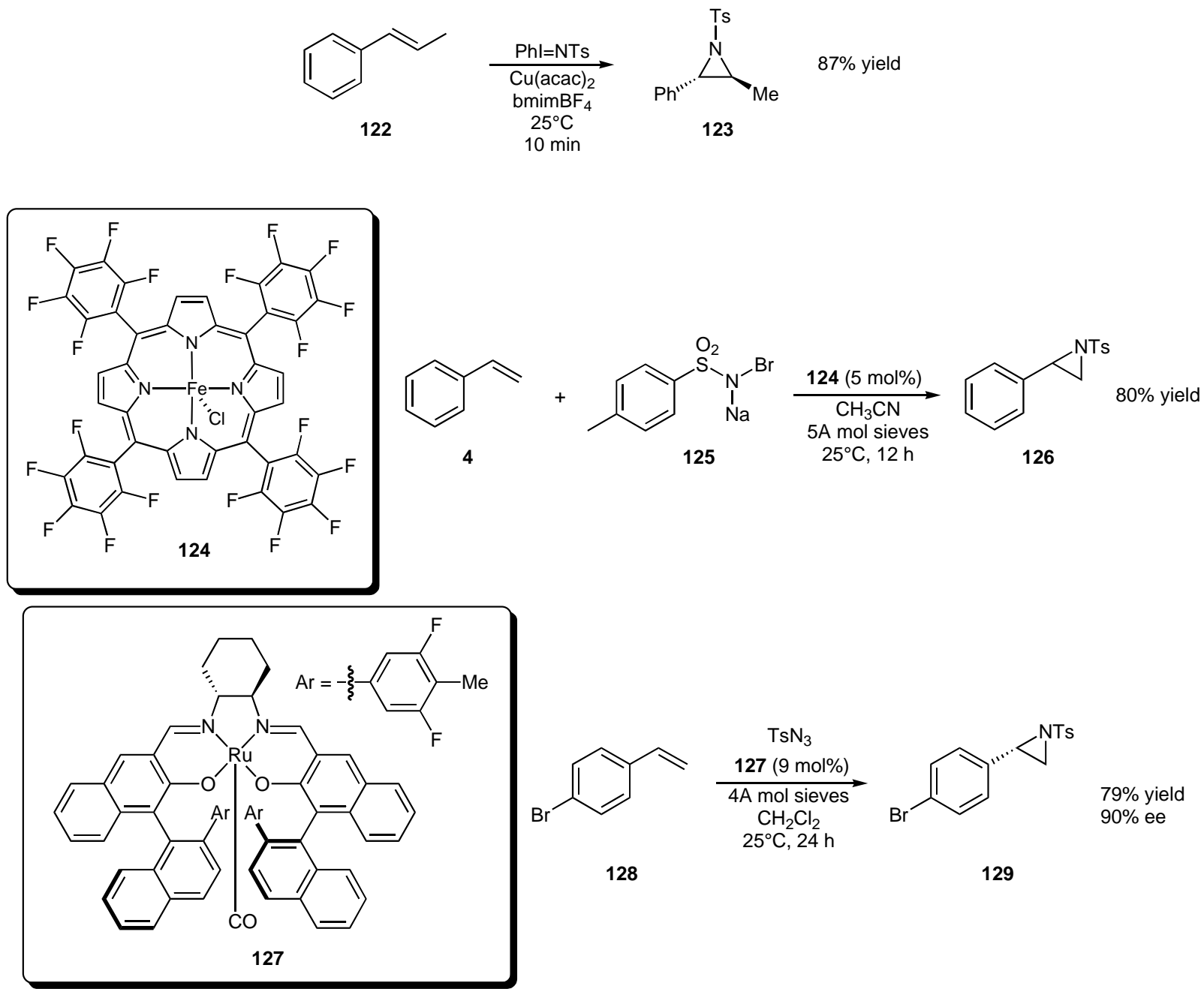

In the case of electron-deficient olefins, other methodologies are also available. For example, the dicyanoalkene $\mathbf{1 3 0}$ (derived from the Knoevenagel condensation of malononitrile with acetaldehyde) undergoes a facile aza-Michael addition of ethyl nosyloxycarbamate in the 
presence of calcium oxide to give a $\beta$-aminocarbanion intermediate (131), which quickly cyclizes to the corresponding dicyanoaziridine (132) in excellent yield. ${ }^{57}$ When trifluoromethylacrylates are used as substrates, the intermediate Michael adducts can be isolated in $>95 \%$ yield. ${ }^{58}$ Some degree of enantioselectivity has been observed when Cinchona alkaloids are used as catalysts in the reaction. ${ }^{59}$ An electrophilic variant is represented by the coppercatalyzed addition of $N, N$-dichloroarylsulfonamide across electron-deficient olefins such as methyl acrylate (133). The resulting $\beta$-chloroamines, which exhibit stereochemistry resulting from net anti addition, can be cyclized by treatment with sodium hydroxide to provide tosyl aziridines (e.g., 135) in good overall yield. ${ }^{60}$

An intriguing electrochemical aziridination is based on the selective anodic oxidation of $\mathrm{N}$ aminophthalimide $(\mathbf{1 3 6}$, oxidation potential $+1.60 \mathrm{~V})$ in the presence of olefins. Thus, trans-hex4-en-3-one (137) is converted to the corresponding aziridine (138) in acetonitrile solution using a platinum electrode at a constant potential of +1.80 volts. The reaction mixture is buffered using triethylammonium acetate, since the cathodic process reduces proton to hydrogen gas. The use of platinum at the anode is critical, as graphite electrodes yielded no aziridination products. ${ }^{61}$
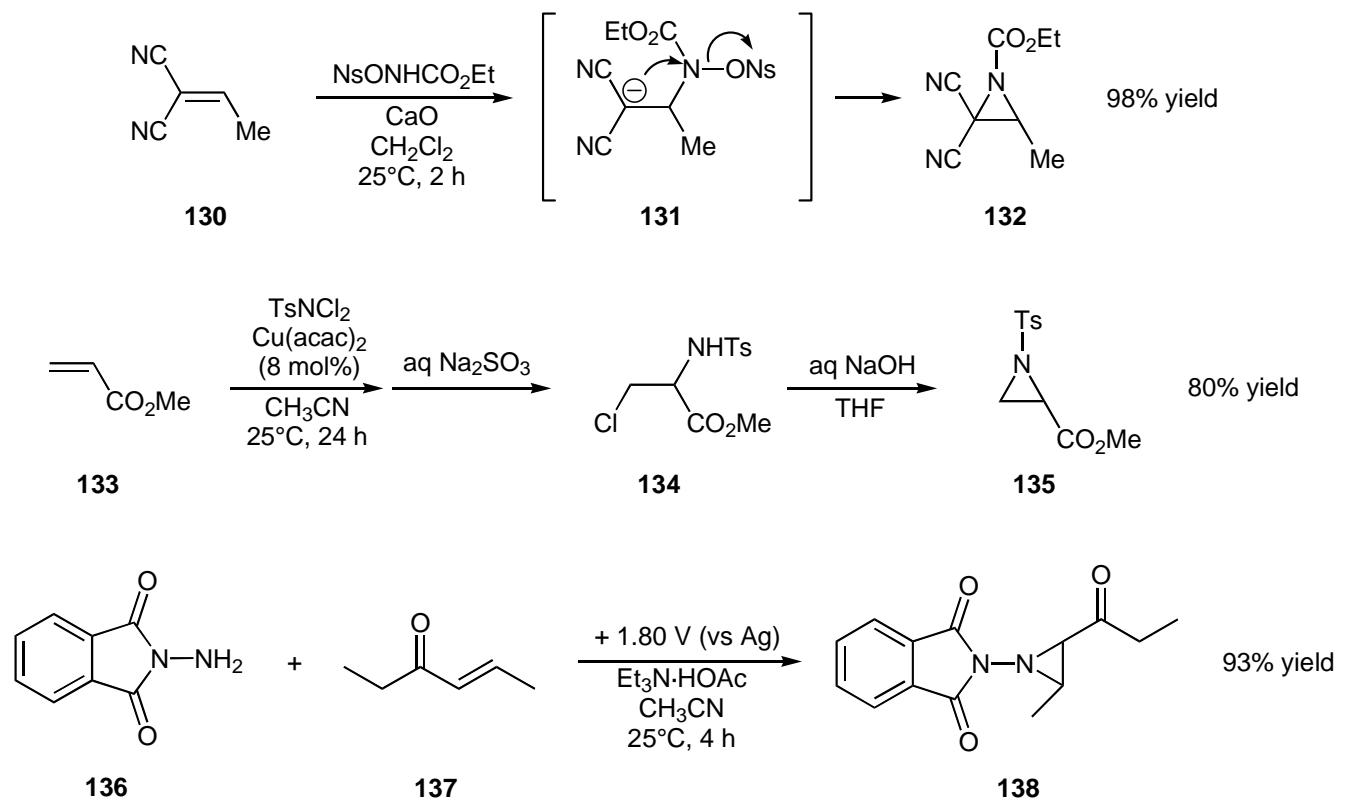

Just as epoxides can be prepared from carbonyl compounds, aziridines can be accessed through the addition of amphophilic carbon centers onto imines, and sulfur ylides are frequently used as carbon donors in this regard. Thus, S-allyl tetrahydrothiophenium bromide (139) is smoothly deprotonated with strong base to provide an ylide which adds to a variety of $N$ protected imines. For the $N$-tosyl aldimine (140) derived from isovaleraldehyde, the corresponding vinyl aziridine (141) is formed in fair yield as a mixture of stereoisomers. 

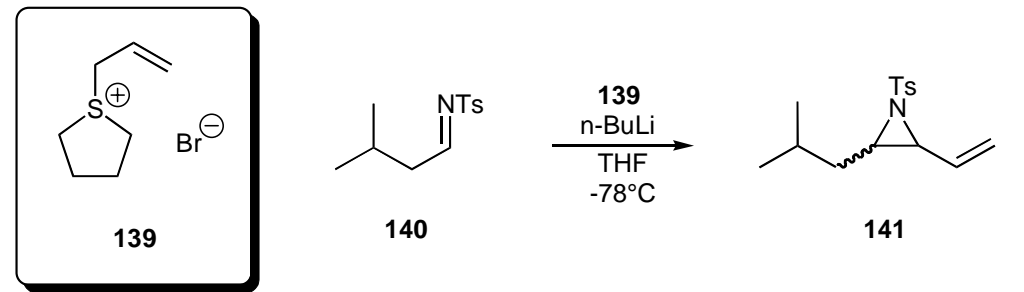

$58 \%$ yield

cis/trans 1:2

When chiral t-butylsulfinylimines (e.g., 142) are used as substrates, a highly stereoselective aziridination ensues, providing the heterocycles in good yield and good to excellent diastereomeric excess. ${ }^{63}$ This approach of leveraging chirality at the imine nitrogen to impart enantioselectivity has also been used to advantage in the preparation of chiral heterosubstituted aziridines. Thus, when 2-(1-chloroethyl)-4-methyl-thiazole (145) is deprotonated with LDA and treated with the chiral aldimine 144, the aziridinyl thiazole derivative 146 is produced in excellent yield and diastereoselectivity.
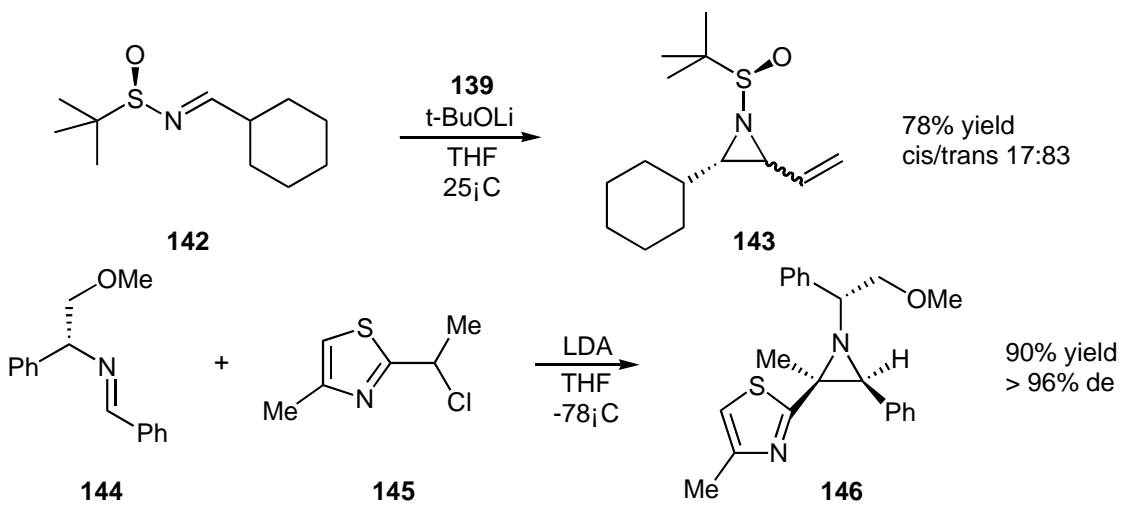

Chiral induction can also be quite effective when the locus of asymmetry is attached to the sulfur ylide itself. The sulfonium salt (147) derived from Eliel's oxathiane can be used to deliver a benzylic center to tosylimines (e.g., 148) and efficiently produces phenylaziridines with a very high degree of asymmetric induction. The method is amenable to gram-quantity synthesis, and the chiral auxiliary can be easily recovered. In general, cis/trans mixtures are obtained, depending upon the steric bulk of the imine substituent.
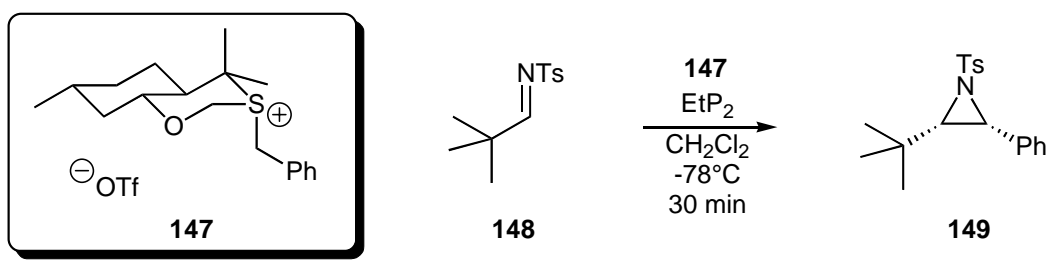

$80 \%$ yield

$98.7 \%$ ee

As a complement to the base-catalyzed ylide approach, the Johnston group has developed a Bronsted acid-catalyzed direct aza-Darzens protocol for the synthesis of $N$-alkylaziridines. Ethyl diazoacetate (151) serves as an acetate enolate synthon under acidic conditions, engaging in 
[2+1] annulation with $N$-alkyl aldimines (e.g., 150) to provide the corresponding aziridine (152) with very high cis-selectivity. The conditions are mild enough that acid-catalyzed ring-opening of the products is not observed. ${ }^{66}$

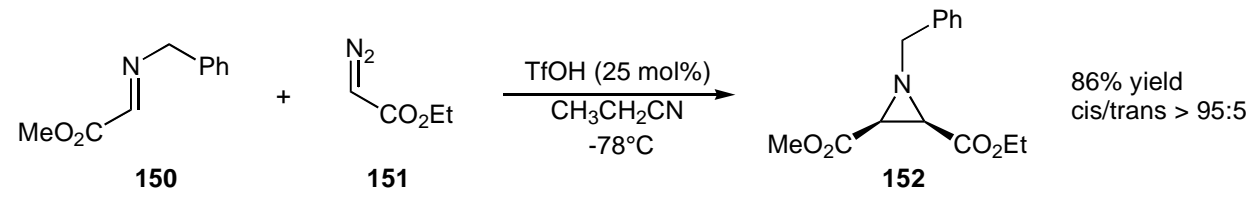

\section{Reactions of Aziridines}

One of the most widely encountered reactions of aziridines is the nucleophilic ring-opening of the heterocyclic ring. This has been the subject of a recent thoroughgoing and excellent review, to which the reader is directed. ${ }^{67}$ The interesting highlights of the previous year can be divided into the two categories of carbon-based nucleophiles and heteroatomic nucleophilic centers. Alkynylation of aziridines can be effected through the copper-catalyzed ring opening with acetylides. For example, lithium phenylacetylide engages in smooth nucleophilic attack of $N$ tosyl-7-azabicyclo[4.1.0] heptane (153) in the presence of copper(I) triflate to provide the cyclohexyl alkyne 154 in excellent yield. ${ }^{68}$ Similarly, the lithium dimethyl cyanocuprate engaged in nucleophilic attack of the less substituted carbon on 1-pentyl- $N$-tosylaziridine 155 to give $N$-tosyl-octane-3-amine (156). In this system, the use of alkyllithium reagents led to eliminative pathways. ${ }^{69}$ However, there are other reports of productive ring-opening by lithiates. Treatment of 6-aza-3-oxabicyclo[3.1.0]hexane (157) with (trimethylsilyl)methyllithium led to the formation of an intermediate ring-opened dianion (158) which subsequently underwent elimination to form the functionalized allylic amine $159 .^{70}$ Key to the success of this protocol is the formation of the aziridinyl anion, which has considerable carbenoid character. A fascinating example of this property is seen in the desymmetrization of the bicyclic tosyl aziridine 160 mediated by s-butyllithium and (-)-sparteine (100). The initially formed anion 161 behaves much like an amino carbene (i.e., 162) which engages in a transannular $\mathrm{C}-\mathrm{H}$ insertion to give the bicyclo[3.3.0] octane derivative 163. Interestingly, the absolute configuration of $\mathbf{1 6 3}$ is the opposite of the alcohol formed from the corresponding rearrangement of the analogous alcohol. ${ }^{71}$
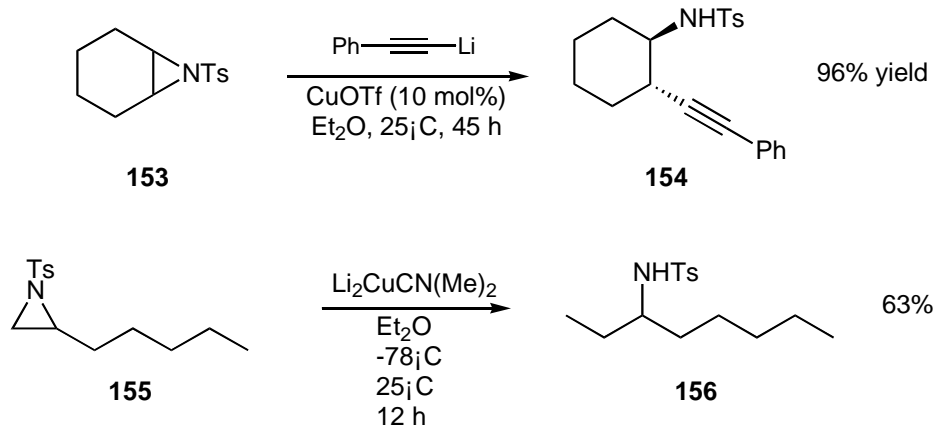

$63 \%$ yield 

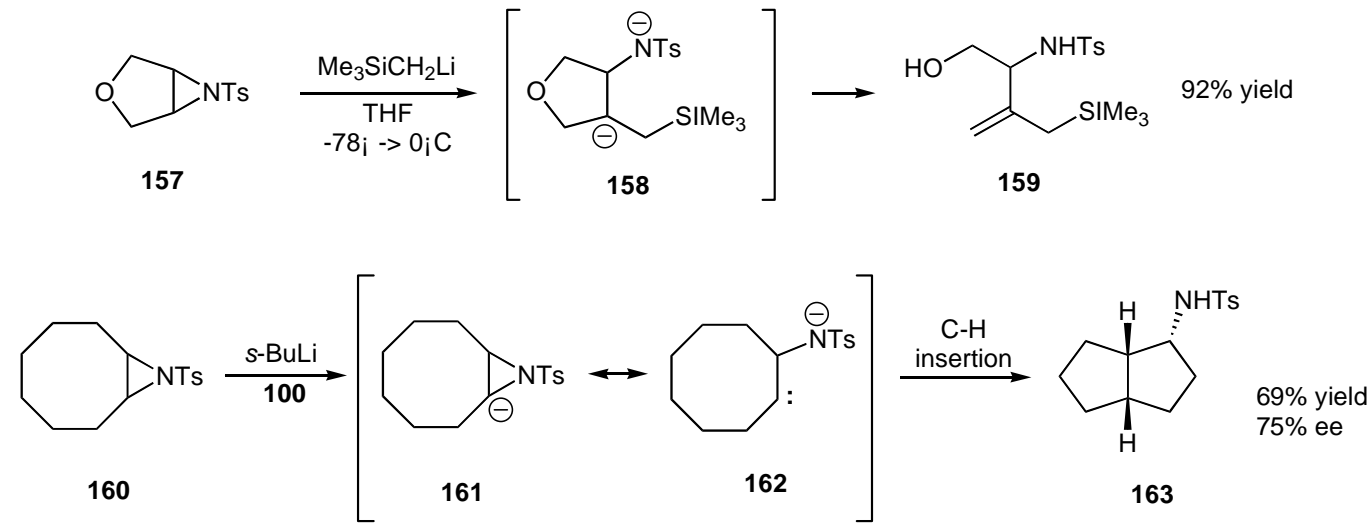

Aziridines can be opened with a variety of heteroatomic nucleophiles. Silica-supported phosphomolybdic acid $\left(\mathrm{PMA}-\mathrm{SiO}_{2}\right)$ is effective in promoting the ring-cleavage of tosyl aziridines by a variety of nucleophiles. Thus, the azide anion preferentially attacked at the benzylic position to provide the azidoaminoalcohol 165, whereby the regioselectivity can be rationalized in terms of partial positive charge stabilization. ${ }^{72}$ A different type of selectivity is observed in the magnesium bromide mediated opening of hydroxymethyl aziridines. In these systems, the delivery of the nucleophile (i.e., bromide) is under chelation control, as demonstrated in the conversion of aziridine 166 to the bromo aminoalcohol $167 .^{73}$ In the absence of Lewis acid catalysts, heteroatomic nucleophiles tend to attack at the sterically least hindered center. Such is the case when the the butylaziridine $\mathbf{1 6 8}$ is treated with tributylphosphine, a strong nonbasic nucleophile which smoothly opens the heterocyclic ring. The initial adduct undergoes rapid proton transfer to provide a betaine (169) which can serve as a Wittig reagent. The addition of benzaldehyde leads to an olefination/elimination sequence to afford the diene $\mathbf{1 7 0}$ as a mixture of $E / Z$ isomers. ${ }^{74}$
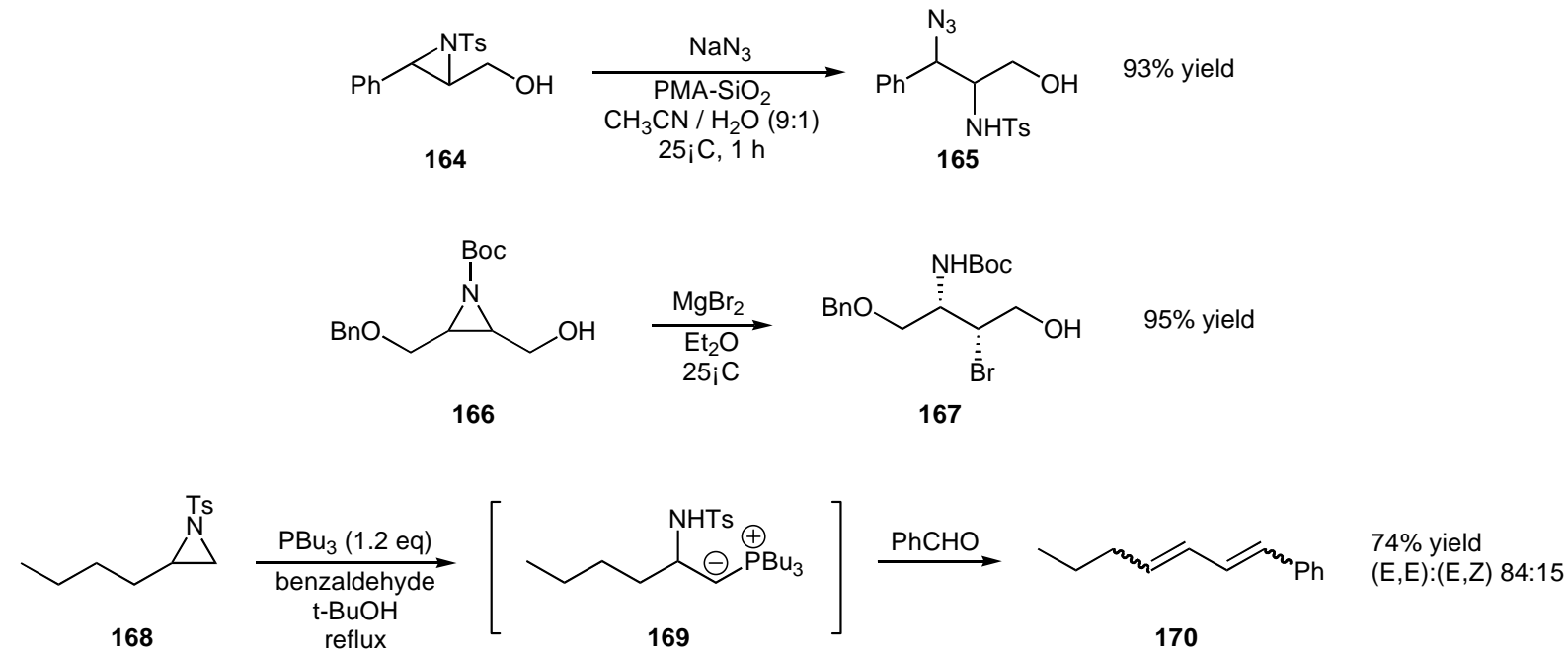
Another interesting and synthetically useful behavior of aziridines is their tendency to open thermally to azomethine ylides, a process which can also be facilitated by Lewis acid catalysts. These reactive intermediates can be trapped by a variety of dipolarophiles to give new heterocyclic species. Methyl vinyl ethers convert aziridines such as $\mathbf{1 7 2}$ into a pyrrolidine derivative (i.e., 174) in the presence of a zinc(salen) Lewis acid catalyst (171). ${ }^{75}$ Similarly, nitriles (e.g., 175) lead to the formation of 2,4-disubstituted $1 H$-imidazolines (e.g., 176) under the catalysis of boron triethyloxonium tetrafluoroborate. ${ }^{76}$ Under almost identical conditions, the aziridine-Lewis acid complex can be trapped with $\pi$-nucleophiles, such as a tethered olefin, to provide fused bicyclic pyrrolidines (e.g., 178). ${ }^{77}$
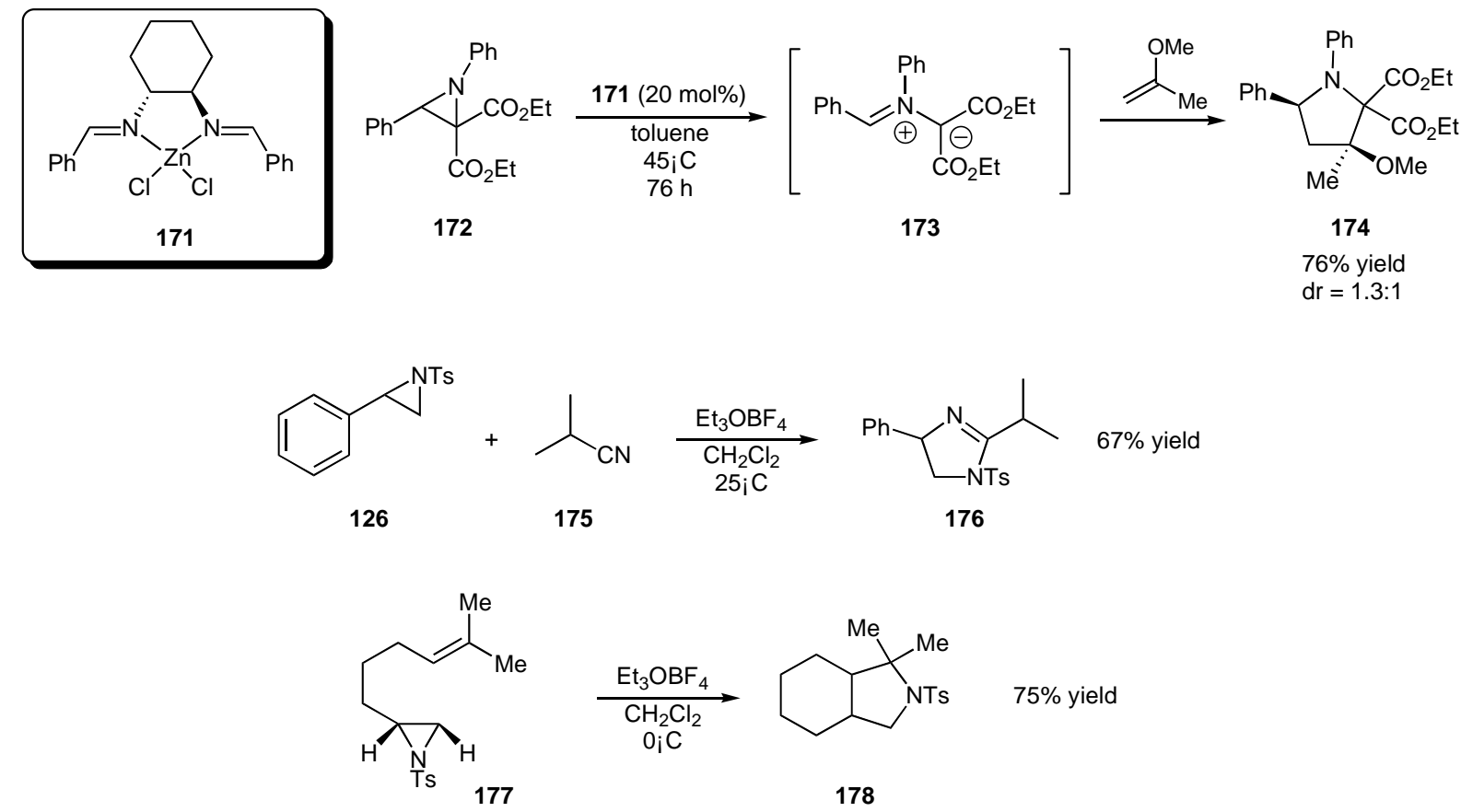

The (salen)chromium complex 179 was shown to promote the insertion of carbon dioxide into aziridines (e.g., 180) to yield the corresponding oxazolidinones (e.g., 181), whereby the substrate is treated with $\mathrm{CO}_{2}$ under high pressure (Parr reactor) in the presence of catalytic quantities of 179 and dimethylaminopyridine (DMAP). ${ }^{78}$ Considerably milder conditions have been reported independently, in which lithium bromide serves as catalyst in a medium of $N$ methylpyrrolidone (NMP). For example, aryl aziridine 182 was converted to oxazolidinone 183 in 79\% yield over 24 hours. Use of the more polar and higher boiling solvent allows for delivery of $\mathrm{CO}_{2}$ using a balloon at atmospheric pressure. Electron-donating substituents tend to accelerate the reaction.

In a similar vein, a resin-supported rhodium-complexed dendrimer (184) has been shown to promote the carbonylative ring expansion of aziridines to $\beta$-lactams, as illustrated by the conversion of the $N$-t-butyl aziridine $\mathbf{1 8 5}$ to the corresponding lactam (186) in almost quantitative yield. The supported catalyst, which shows reactivity comparable to the solution- 
phase variety, is easily recovered by filtration and exhibits no significant loss of activity upon recycling.

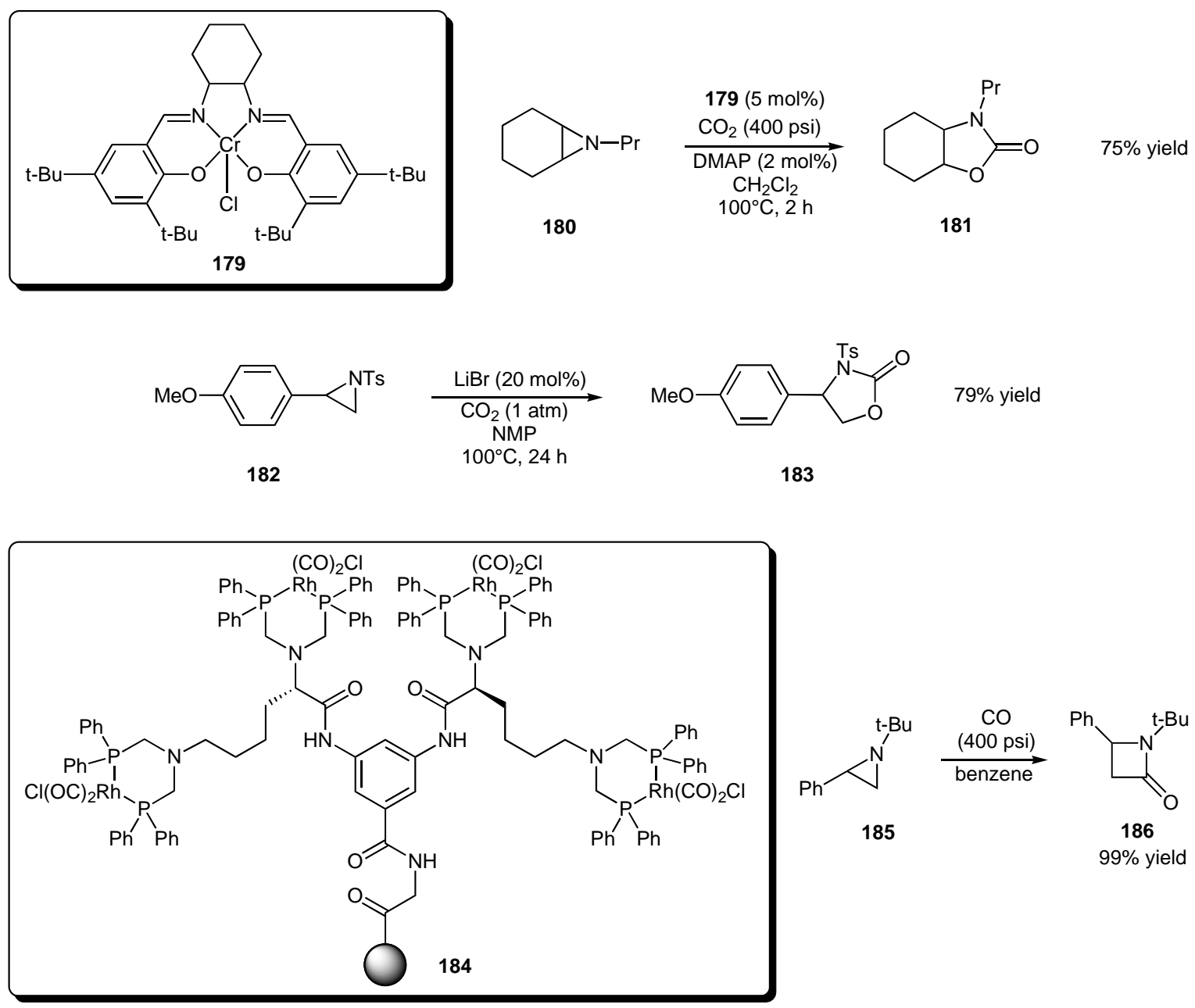

\section{References}

1. Ramón, D. J.; Yus, M. Curr. Org. Chem. 2004, 8, 149.

2. Abashkin, Y. G.; Burt, S. K.; Org. Lett. 2004, 6, 59.

3. Bhattacharjee, S.; Anderson, J. A.; J. Chem. Soc., Chem. Commun. 2004, 554.

4. Wentzel, B. B.; Alsters, P. L.; Feiters, M. C.; Nolte, R. J. M. J. Org. Chem. 2004, 69, 3453.

5. Farinas, E. T.; Alcalde, M.; Arnold, F. Tetrahedron, 2004, 60, 525.

6. Chan, W. -K.; Liu, P.; Yu, W. -Y.; Wong, M. -K.; Che, C. -M. Org. Lett. 2004, 6, 1597.

7. Rebelo, S. L. H.; Simões, M. M. Q.; Graça P. M. S. Neves, M.; Silva, A. M. S.; Cavaleiro, J. A. S. J. Chem. Soc., Chem. Commun. 2004, 608.

8. Wang, S. H.; Mandimutsira, B. S.; Todd, R.; Ramdhanie, B.; Fox, J. P.; Goldberg, D. P. J. Am. Chem. Soc. 2004, 126, 18. 
9. Murphy, A.; Pace, A.; Stack, T. D. P. Org. Lett. 2004, 6, 3119.

10. Liu, Y.; Murata, K.; Inaba, M. J. Chem. Soc., Chem. Commun. 2004, 582.

11. Deubel, D. V.; Frenking, G.; Gisdakis, P.; Herrmann, W. A.; Rösch, N.; Sundermeyer, J. Acc. Chem. Res. 2004, 37, 645.

12. Kan, J. T. W.; Toy, P. H. Tetrahedron Lett. 2004, 45, 6357.

13. Shi, Y. Acc. Chem. Res. 2004, 37, 488.

14. Yang, D. Acc. Chem. Res. 2004, 37, 497.

15. Bulman Page, P. C.; Buckley, B. R.; Blacker, A. J. Org. Lett. 2004, 6, 1543.

16. Bulman Page, P. C.; Barros, D.; Buckley, B. R.; Ardakani, A.; Marples, B. A. J. Org. Chem. 2004, 69, 3595.

17. Armstrong, A. Angew. Chem., Int. Ed. 2004, 43, 1460.

18. Ooi, T.; Ohara, D.; Tamura, M.; Maruoka, K. J. Am. Chem. Soc. 2004, 126, 6844.

19. Lopez-Pedrosa, J. -M.; Pitts, M. R.; Roberts, S. M.; Saminathan, S.; Whittall, J. Tetrahedron Lett. 2004, 45, 5073.

20. Geller, T.; Krüger, C. M.; Militzer, H. -C. Tetrahedron Lett. 2004, 45, 5069.

21. Rodríguez, S.; Vidal, A.; Monroig, J. J.; González, F. V. Tetrahedron Lett. 2004, 45, 5359.

22. Aggarwal, V. K.; Winn, C. L. Acc. Chem. Res. 2004, 37, 611.

23. Bellenie, B. R.; Goodman, J. M. J. Chem. Soc., Chem. Commun. 2004, 1076.

24. Arai, S.; Tokumaru, K.; Aoyama, T. Tetrahedron Lett. 2004, 45, 1845.

25. Muthusamy, S.; Gunanathan, C.; Nethaji, M. Synlett, 2004, 639.

26. Nielsen, L. P. C.; Stevenson, C. P.; Blackmond, D. G.; Jacobsen, E. N. J. Am. Chem. Soc., 2004, 126, 1360.

27. Zhao, L.; Han, B.; Huang, Z.; Miller, M.; Huang, H.; Malashock, D. S.; Zhu, Z.; Milan, A.; Robertson, D. E.; Weiner, D. P.; Burk, M. J. J. Am. Chem. Soc. 2004, 126, 11156.

28. Yu, X. -Q.; Hirai, A.; Miyashita, M. Chem. Lett. 2004, 33, 764.

29. Huerta, G. Contreras-Ordoñez, G.; Alvarez-Toledano, C.; Santes, V.; Gómez, E.; Toscano, R. A. Synth. Commun. 2004, 34, 2393.

30. Khosropour, A. R.; Khodaei, M. M.; Ghozati, K. Chem. Lett. 2004, 33, 304.

31. Chakraborti, A. K.; Rudrawar, S.; Kondaskar, A. Eur. J. Org. Chem. 2004, 3597.

32. Zhao, P. -Q.; Xu, L. -W.; Xia, C. -G. Synlett 2004, 846.

33. Rodríguez, J. R.; Navarro, A. Tetrahedron Lett. 2004, 45, 7495.

34. Ollevier, T.; Lavie-Coupin, G. Tetrahedron Lett. 2004, 45, 49.

35. Carrée, F.; Gil, R.; Collin, J. Tetrahedron Lett. 2004, 45, 7749.

36. Bartoli, G.; Bosco, M.; Carlone, A.; Locatelli, M.; Massaccesi, M.; Melchiorre, P.; Sambri, L. Org. Lett. 2004, 6, 2173.

37. Boruwa, J.; Borah, J. C.; Kalita, B.; Barua, N. C. Tetrahedron Lett. 2004, 45, 7355.

38. Ha, J. D. ; Shin, E. Y.; Kang, S. K.; Ahn, J. H.; Choi, J. -K. Tetrahedron Lett. 2004, 45, 4193.

39. Gil, S.; Torres, M.; Ortúzar, N.; Wincewicz, R.; Parra, M. Eur. J. Org. Chem. 2004, 2160.

40. Maslak, V.; Matovic, R.; Saicic, R. N. Tetrahedron, 2004, 60, 8957. 
41. Ciaccio, J. A.; Smrtka, M.; Maio, W. A.; Rucando, D. Tetrahedron Lett. 2004, 45, 7201.

42. Hodgson, D. M.; Fleming, M. J.; Stanway, S. J. J. Am. Chem. Soc., 2004, 126, 12250.

43. Hodgson, D. M.; Chung, Y. K.; Paris, J. -M. J. Am. Chem. Soc. 2004, 126, 8664.

44. Denichoux, A.; Ferreira, F.; Chemla, F. Org. Lett. 2004, 6, 3509.

45. Hodgson, D. M.; Reynolds, N. J.; Coote, S. J. Org. Lett. 2004, 6, 4187.

46. Hodgson, D. M.; Kirton, E. H. M. Synlett 2004, 1610.

47. Dake, G. R.; Fenster, M. D. B.; Fleury, M.; Patrick, B. O. J. Org. Chem. 2004, 69, 5676.

48. Chen, J.; Che, C. -M. Angew. Chem., Int. Ed. 2004, 43, 4950.

49. Ming-Yuan, L.; Madhushaw, R. J.; Liu, R. -S. J. Org. Chem. 2004, 69, 7700.

50. Madhushaw, R. J.; Lin, M. -Y.; Md. Abu Sohel, S.; Liu, R. -S. J. Am. Chem. Soc. 2004, 126, 6895.

51. Kwong, H. -L.; Liu, D.; Chan, K. -Y.; Lee, C. -S.; Huang, K. -H.; Che, C. -M. Tetrahedron Lett. 2004, 45, 3965.

52. Han, H.; Bae, I.; Yoo, E. J.; Lee, J.; Do, Y.; Chang, S. Org. Lett. 2004, 6, 4109.

53. Padwa, A.; Flick, A. C.; Leverett, C. A.; Stengel, T. J. Org. Chem. 2004, 69, 6377.

54. Kantam, M. L.; Neeraja, V.; Kavita, B.; Haritha, Y. Synlett 2004, 525.

55. Vyas, R.; Gao, G. -Y.; Harden, J. D.; Zhang, X. P. Org. Lett. 2004, 6, 1907.

56. Omura, K.; Uchida, T.; Irie, R.; Katsuki, T. J. Chem. Soc., Chem. Commun. 2004, 2060.

57. Fioravanti, S.; Morreale, A.; Pellacani, L.; Tardella, P. A. Synlett 2004, 1083.

58. Colantoni, D.; Fioravanti, S.; Pellacani, L.; Tardella, P. A. Org. Lett. 2004, 6, 197.

59. Fioravanti, S.; Gabriella Mascia, M.; Pellacani, L.; Tardella, P. A. Tetrahedron 2004, 60, 8073.

60. Nadir, U. K.; Singh, A. Synth. Commun. 2004, 34, 1337.

61. Caiazzo, A.; Dalili, S.; Picard, C.; Sasaki, M.; Siu, T.; Yudin, A. K. Pure Appl. Chem. 2004, $76,603$.

62. Arini, L. G.; Sinclair, A.; Szeto, P.; Stockman, R. A. Tetrahedron Lett. 2004, 45, 1589.

63. Morton, D.; Pearson, D.; Field, R. A.; Stockman, R. A. Org. Lett. 2004, 6, 2377.

64. De Vitis, L.; Florio, S.; Granito, C.; Ronzini, L.; Troisi, L.; Capriati, V.; Luisi, R.; Pilati, T. Tetrahedron 2004, 60, 1175.

65. Solladié-Cavallo, A.; Roje, M.; Welter, R.; Sunjic, V. J. Org. Chem. 2004, 69, 1409.

66. Williams, A.L.; Johnston, J. N. J. Am. Chem. Soc. 2004, 126, 1612.

67. Hu, X. E. Tetrahedron 2004, 60, 2701.

68. Ding, C. -H.; Dai, L. -X.; Hou, X. -L. Synlett 2004, 1691.

69. D'hooghe, M.; Kerkaert, I.; Rottiers, M.; De Kimpe, N. Tetrahedron 2004, 60, 3637.

70. Hodgson, D. M.; Stefane, B.; Miles, T. J.; Witherington, J. J. Chem. Soc., Chem. Commun. 2004, 2234.

71. Müller, P.; Riegert, D.; Bernardinelli, G. Helv. Chim. Acta 2004, 87, 227.

72. Kishore Kumar, G. D.; Baskaran, S. Synlett 2004, 1719.

73. Righi, G.; Catullo, S. Synth. Commun. 2004, 34, 85.

74. Fan, R. -H.; Hou, X. -L.; Dai, L. -X. J. Org. Chem. 2004, 69, 689. 
75. Pohlhaus, P. D.; Bowman, R. K.; Johnson, J. S. J. Am. Chem. Soc. 2004, 126, 2294.

76. Bhanu Prasad, B. A.; Pandey, G.; Singh, V. K. Tetrahedron Lett. 2004, 45, 1137.

77. Bergmeier, S. C.; Katz, S. J.; Huang, J.; McPherson, H.; Donoghue, P. J.; Reed, D. D. Tetrahedron Lett. 2004, 45, 5011.

78. Miller, A. W.; Nguyen, S. T. Org. Lett. 2004, 6, 2301.

79. Sudo, A.; Morioka, Y.; Sanda, F.; Endo, T. Tetrahedron Lett. 2004, 45, 1363.

\section{Authors' biographical data}

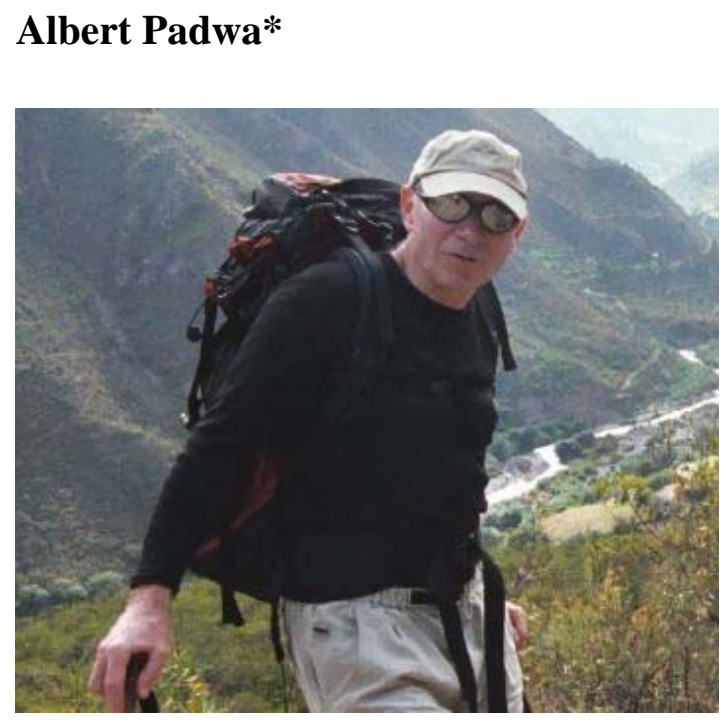

Albert Padwa was born in New York City. He received both his B.A. and Ph.D. degrees from Columbia University. After a NSF postdoctoral position at the University of Wisconsin, he was appointed Assistant Professor of Chemistry at the Ohio State University in 1963. He moved to SUNY Buffalo in 1966 as Associate Professor and was promoted to Professor in 1969. Since 1979, he has been the William Patterson Timmie Professor of Chemistry at Emory University. He has held visiting positions at University Claude Bernard, France, University of California at Berkeley, the University of Wurzburg, Germany, Imperial College of Chemistry, UK and the University of Melbourne, Australia. Professor Padwa has been the recipient of an Alfred P. Sloan Fellowship, John S. Guggenheim Fellowship, Alexander von Humboldt Senior Scientist Award, a Fulbright Hays Scholarship, Senior Award in Heterocyclic Chemistry from the International Society of Heterocyclic Chemists, ACS Arthur C. Cope Scholar Award and is the coauthor of more than 600 publications. He served as the Chairman of the Organic Division of the ACS and as President of the International Society of Heterocyclic Chemistry. He has also served as a member of the editorial boards of the Journal of the American Chemical Society, Journal of Organic Chemistry, Organic Letters, and has been the volume editor of Comprehensive Heterocyclic Chemistry, the Synthesis of Science (Vol 27) and is currently one 
of the Associate Editors of the Journal of Organic Chemistry. His research interests include heterocyclic chemistry, dipolar cycloadditions, alkaloid synthesis, tandem transformations, organometallic chemistry, and organic photochemistry. Aside from chemistry, his other passion is mountain climbing in various parts of the world.

* Author to whom inquiries concerning this article should be sent

\section{S. Shaun Murphree}

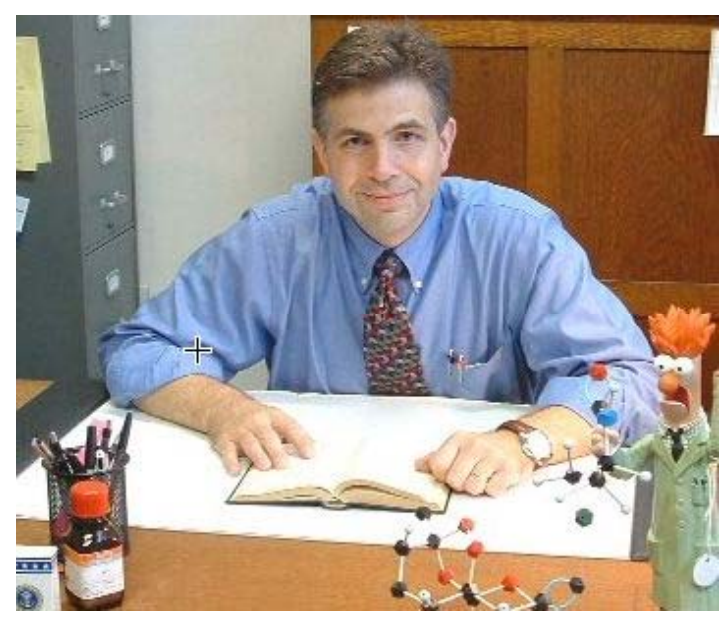

S. Shaun Murphree received his B. A. degree from Colgate University in 1984, and earned his Ph.D. from Emory University in 1991 under the supervision of Professor Albert Padwa. After a postdoctoral fellowship in the laboratories of Professor Peter Jacobi at Wesleyan University in 1992, he accepted a position as research chemist at Bayer Corporation in Charleston, S. C. He joined the faculty at Allegheny College in 1999. His research interests focuses mainly on synthetic methodology, particularly through the use of sulfones. An additional research interest involves the development of more environmentally benign modifications of classical industrial syntheses through the use of catalytic methods. 